\section{Ein Rechenmodell zur Beschreibung der Förderung pastöser Stoffe in einer Dekantierzentrifuge*}

\section{Andreas Karolis und Werner Stahl**}

Bei der Verarbeitung feinstdisperser Suspensionen in Dekantierzentrifugen können beim Transport des absedimentierten Feststoffs im konischen Teil der Maschine erhebliche Probleme auftreten. Während grobkörnige Feststoffe mit hohem innerem Reibwert im allgemeinen problemlos von der Schnecke des Dekanters aus dem zylindrischen über den konischen Teil des Rotors gefördert und ausgetragen werden, kommt es bei Sedimenten mit pastöser, schlammartiger Konsistenz zu einem Rückstau. Solche Stoffe widersetzen sich der Hochförderung durch die Schnecke; sie fließen konusabwärts in den zylindrischen Teil der Zentrifuge zurück und beeinträchtigen damit das Klärverhalten des Dekanters. Das Sediment kann entgegen seiner Förderrichtung sowohl durch den Spalt zwischen Schneckenblatt und " nuswand als auch spiralig entlang des Schneckenkanals zurückflie*..n [1].

Für den Fall, daß das Sediment nur in spiraliger Richtung entlang des Schneckenkanals zurückfließen kann (bei fester Grundschicht), ist ein Rechenmodell entwickelt worden, mit dem es möglich ist, in Abhängigkeit von der Maschinengeometrie, der Betriebseinstellung und den rheologischen Eigenschaften der Paste Aussagen über die Förderfähigkeit bzw. über den möglichen Rückstau des Schlammes zu treffen. Die aus den Impulsgleichungen und dem rheologischen Reibungsansatz für nicht-Newtonsche Medien [2] abgeleitete Transportgleichung lautet:

$\dot{m}_{\text {Zulauf }}=\bar{\rho}(1-\mathrm{RF})\left\{2 \pi R h(G-b) n_{\text {Diff }} \cos ^{2} \alpha-\right.$

$-(G-b) \cos \alpha \frac{n}{n+1} K^{-1 / n}\left(4 \pi^{2} \bar{\rho} R n_{\mathrm{H}}^{2} \sin \alpha \cos \beta\right)^{1 / n} \times$

$\left.\times\left(\frac{\mathrm{d} h}{\mathrm{~d} l}-\tan \beta\right)^{1 / n}\left(-\left(\frac{h-z_{0}}{\cos \delta}\right)\right)^{\frac{2 n+1}{n}}\left[\left(\frac{n+1}{2 n+1}\right)+\left(\frac{z_{0}}{h-z_{0}}\right)\right]\right\}+\dot{m}_{\text {Zentrat }}$

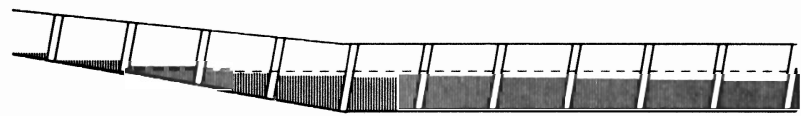

Maschinentyp A

Zylinderdurcnmesser $D_{z y 1}=250 \mathrm{~mm}$ Austragsdurchmesser $D_{\text {Austr }}=165 \mathrm{~mm}$ Niveaudurchmesser $\quad D_{\text {wiv }}=190 \mathrm{~mm}$ Zylinder länge $\quad L_{2 y 1}=340 \mathrm{~mm}$ Konuswinkel $\quad B=10 \mathrm{Grad}$ Gangnőne $\quad G=55 \mathrm{~mm}$ Blattstärke $\quad b=6 \mathrm{~mm}$ Niveauvolumen $\quad V_{\text {wiv }}=7.7 \mathrm{dm}^{3}$
Abb. 1. Berechnetes Rückstauprofil; der Start erfolgt am Austragsradius.

* Vortrag von A. Karolis auf dem Jahrestreffen der VerfahrensIngenieure, 25. bis 27. Sept. 1985 in Hamburg.

** Dipl.-Ing. A. Karolis und Prof. Dr.-Ing. W. Stahl, Institut für Mechanische Verfahrenstechnik und Mechanik der Univ. Karlsruhe (TH), Kaiserstr. 12, 7500 Karlsruhe 1.
( $\dot{m}_{\text {Zulauf }}$ Feststoffmassenstrom im Zulauf, $\bar{\rho}$ mittlere Sedimentsdichte, RF Restfeuchte, $R$ Radius, $h$ Kuchenhöhe, $G$ Ganghöhe, $b$ Blattdikke, $n_{\text {Diff }}$ Differenzdrehzahl, $\alpha$ Schneckensteigungswinkel, $n$ Fließindex, $K$ Fließkonstante, $n_{\mathrm{H}}$ Rotordrehzahl, $\beta$ Konuswinkel, $l$ Ortskoordinate parallel zur Drehachse der Zentrifuge, $\delta$ Verschneidungswinkel, $z_{0}$ ungescherter Anteil der Kuchenhöhe, $\dot{m}_{\text {Zentrat }}$ Feststoffmassenstrom im Zentrat).

Bei Gl. (1) handelt es sich um eine gewöhnliche Differentialgleichung höherer Ordnung, die analytisch nicht lösbar ist. Bei der numerischen Vorgehensweise zur Berechnung des Rückstauprofils über die gesam-

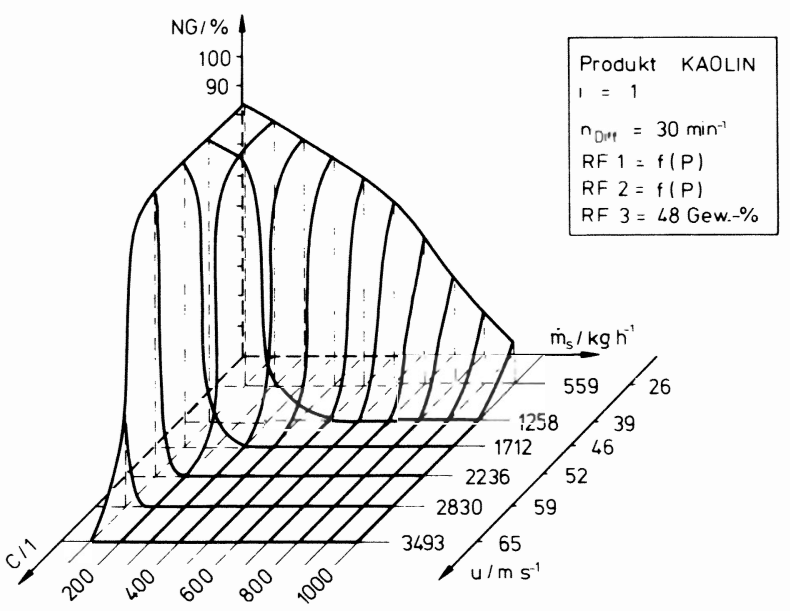

Abb. 2. Betriebskennfeld zur Beurteilung der Funktionsfähigkeit des Dekanters.

te Dekanterlänge wird - ausgehend vom Austragsradius als Startpunkt - iterativ konusabwärts gerechnet. Abb. 1 zeigt ein berechnetes Rückstauprofil für eine Maschine bestimmter Geometrie und Betriebseinstellung.

Es werden dabei zwei Begriffe eingeführt, mit denen die Funktionsfähigkeit des Dekanters besser beurteilt werden kann:

a) Nutzungsgrad NG: Er ist ein Maß für das noch zur Verfügung stehende Klärvolumen und wird wie folgt definiert:

$$
\mathrm{NG}=\left(1-\frac{\mathrm{VS} 1}{\mathrm{VNIV}}\right) 100[\%]
$$

wobei VNIV das Niveauvolumen und VS1 das Schlammvolumen ist, welches das Niveauvolumen verdrängt.

b) Füllgrad FG: Damit kann beurteilt werden, um welchen Faktor das Schlammvolumen im Dekanter gegenüber dem rückstaufreien Fall größer ist. Er ist ein Maß für die tatsächliche Verweilzeit des Sediments in der Maschine und ist definiert als das Verhältnis aus dem gesamten Schlammvolumen mit Rückstau (VS) bezogen auf das gesamte Schlammvolumen ohne Rückstau (VK):

$\mathrm{FG}=\mathrm{VS} / \mathrm{VK} \geq 1$.

In Abb. 1 ist RF1 die Restfeuchte am Feststoffaustrag, RF2 jene am Übergang Konus/Zylinder und RF3 die Restfeuchte am Zylinderende, wo die Suspension im Falle eines Gleichstromdekanters aufgegeben wird.

In dem oben gezeigten Beispiel zur Berechnung des Rückstauprofils (Abb. 1) kann man ersehen, daß nur 19,2\% des gesamten Niveauvolumens für die Klärung zur Verfügung stehen. Für dieses Beispiel würde sich der Feststoff 4,6mal länger in der Zentrifuge aufhalten, als wenn diese rückstaufrei funktionieren würde. Setzt man nun die Rotordrehzahl von $3000 \mathrm{~min}^{-1}$ auf $2750 \mathrm{~min}^{-1}$ herab, so steigt der Nutzungsgrad von $19,2 \%$ auf $73,4 \%$ an. Den Nutzungsgrad kann man 


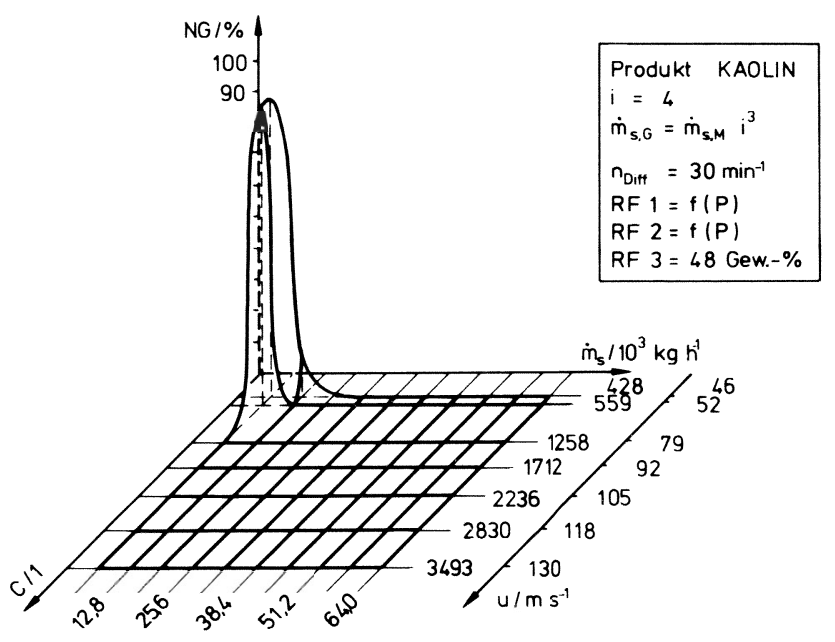

Abb. 3. Betriebskennfeld beim Scale-up.

auch verbessern, indem man die Rotordrehzahl konstant hält, dafür aber die Differenzdrehzahl erhöht. Eine Erhöhung der Differenzdrehzahl z. B. von $30 \mathrm{~min}^{-1}$ auf $35 \mathrm{~min}^{-1}$ würde zu einer Verbesserung des Nutzungsgrades von $19,2 \%$ auf $53,3 \%$ führen. Bei solchen Überlegungen muß man natürlich bedenken, daß dabei andere unerwünschte Effekte auftreten können, so z. B. Störung der Klärung durch Turbulenzen bei Erhöhung der Differenzdrehzahl bzw. verminderte Sedimentationswirkung und höhere Restfeuchten beim Herabsetzen der Rotordrehzahl.

Die oben erwähnten Beispiele sind einzelne Betriebszustände. Trägt man nun den Nutzungsgrad NG in Abhängigkeit von der Schleuderziffer $C$ und dem Feststoffdurchsatz $\dot{m}_{\mathrm{s}}$ auf (Abb. 2), so kann man ersehen, bei welchen Betriebskombinationen der Dekanter noch relativ störungsfrei funktioniert.

Interessant wird es, wenn man das Ergebnis aus einem Pilot-Versuch auf eine Großmaschine überträgt (Scale-up). Dabei werden die einzelnen Daten folgendermaßen verändert:

Modellausführung (M)

$C_{\mathrm{M}}$

$n_{\text {Diff,M }}$

$\dot{m}_{\text {Zulauf,M }}$

$G_{\mathrm{M}}$

$b_{\mathrm{M}}$

$\beta_{\mathrm{M}}$

$D_{\mathrm{Zyl}, \mathrm{M}}$

$D_{\text {Austrag,M }}$

$D_{\mathrm{Niv}, \mathrm{M}}$

Vergrößert man die Pilot-Maschine (Abb. 1) z. B. auf das vierfache (Stufensprung $i=4$ ), so sinkt der Nutzungsgrad für die Großmaschine auf Null. Abb. 3 zeigt, daß der Einsatz eines Großdekanters praktisch nicht sinnvoll ist, wenn beim Scale-up die Schleuderziffer $C$ konstant gehalten wird. Die Großmaschine würde nur noch in einem sehr engen Bereich rückstaufrei funktionieren.

Vergleicht man Abb. 3 mit Abb. 2, so erkennt man, daß die Großmaschine bezüglich des Rückstaus sich ähnlich verhalten würde, wenn beim Scale-up nicht die Schleuderziffer $C$, sondern die Umfangsgeschwindigkeit $u$ der Trommel konstant gehalten würde. Dies kann auch durch Ähnlichkeitsbetrachtung aus Gl. (1) abgeleitet werden. Für die Schleuderziffer gilt demnach: $C_{\mathrm{G}}=C_{\mathrm{M}} / i$. Die Großmaschine wird bei konstanter Umfangsgeschwindigkeit sogar günstiger als die Pilot-Maschine funktionieren, wenn man beim Scale-up den Feststoffdurchsatz $\dot{m}_{\mathrm{s}}$ nicht nach dem Schleppkraftansatz [3] mit $i^{3}$, sondern nach der Theorie der äquivalenten Klärfläche mit $i^{2}$ vergröBert.

Wie schon erwähnt, wurden die hier aufgeführten Beispiele unter der

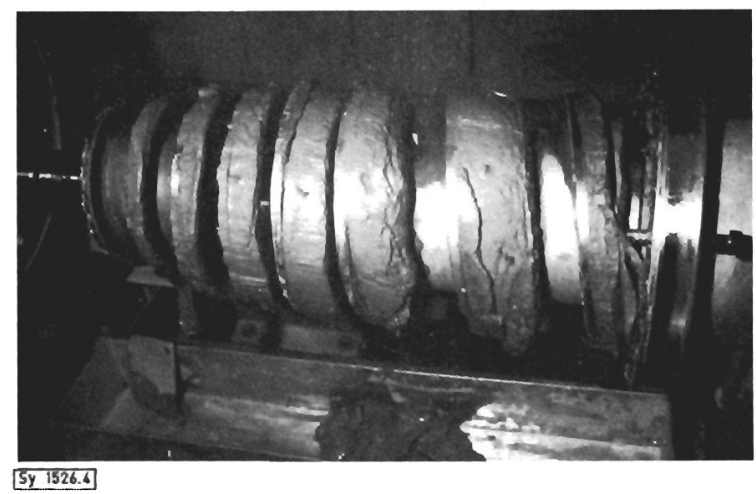

Abb. 4. Schlammrückstau in einem ausgebauten Dekanter. (Durch das Herausziehen der Schnecke streift sich der deponierte Feststoff ebenso wie die Grundschicht auf der Rückseite der Schneckenblätter ab. Die Formation des Kuchens entspricht deshalb nicht dem Zustand im Betrieb.)

Annahme einer festen Grundschicht berechnet. Für den Fall, daß das Sediment neben der spiraligen Rückströmung auch durch den Spalt zwischen Schneckenblatt und Konuswand zurückfließen kann, wird das Rückstauprofil noch ungünstiger. Hinzu kommt, daß bei gleichzeitigem Rückfließen des Schlamms durch den Spalt und in spiraliger Richtung - je nach Oberflächenrauhigkeit - auch das Gleiten der Paste an den Metalloberflächen berücksichtigt werden muß.

Bei der Herleitung dieses Rechenmodells bzw. bei der Berechnung der Rückstauprofile wurden gewisse Annahmen getroffen, wodurch mit entsprechender Vorsicht auch quantitative Aussagen gemacht werden können. Mit dem Rechenmodell ist man jetzt besser in der Lage, Relativaussagen zu treffen, vor allem dann, wenn man das Versuchsergebnis einer Pilot-Maschine auf eine Großmaschine übertragen möchte. Kritisch zu sehen ist der große experimentelle Aufwand zur Bestimmung der rheologischen Daten $\left(\tau_{0}, K, n\right)$, die von der Restfeuchte des Sediments abhängen, welche sich über den Transportweg fortlaufend ändert. Da diese Änderung der Schlammrestfeuchte während der Schneckenförderung nicht bekannt ist, wurde sie in erster Näherung als linear angenommen. Man könnte jedoch auch davon ausgehen, daß sich die Restfeuchte im konischen Bereich des Dekanters durch die Abnahme der Zentrifugalbeschleunigung nicht oder kaum mehr ändert, so daß die Werte für RF1 und RF2 für die Rechnung praktisch konstant gehalten werden können.

Das hier beschriebene Rechenmodell wurde auch anhand experimenteller Daten aus einem industriellen Einsatzfall überprüft. Bei diesem Einsatzfall wurden zur Behandlung von Stärke-Suspension zwei Dekanter unterschiedlicher Geometrie eingesetzt. Dabei hat man festgestellt, daß die größere Maschine den Feststoff überhaupt nicht austrug. Nach Ermittlung der rheologischen Daten des Produkts konnte dieser experimentelle Befund auch rechnerisch nachgewiesen werden.

Eingegangen am 31. Oktober 1985

[1] Karolis, A.; Stahl, W.: Proc. Symp. Solids/Liquids Separation Practice and the Influence of New Techniques, Univ. of Leeds, England, 2.-5. April 1984.

[2] Windhab, E.; Gleißle, W.: Advances in Rheology, Vol. 2: Fluids, Proc. IX. Int. Congress on Rheology, Mexico 1984, p. 557/ 564.

[3] Stahl, W.; Langeloh, Th.: Chem.-Ing.-Tech. 55 (1983) S. 324/325; Ger. Chem. Eng. 7 (1984) S. 72/84.

Schlüsselworte: Dekantierzentrifuge, pastöse Förderung, Rückstau, Störung der Klärung, Rheologie, Scale-up.

Das vollständige Manuskript dieser Arbeit umfaßt 29 Seiten mit 17 Abbildungen, 3 Tabellen und 3 Literaturzitaten. Es ist als Fotokopie oder Mikrofiche MS 1456/86 erhältlich. Eine Bestellkarte finden Sie am Schluß dieses Heftes. 
Ein Rechenmodell zur Beschreibung der Förderung pastöser Stoffe in einer Dekantierzentrifuge *)

Andreas Karolis und Werner Stahl **)

\section{$\underline{\text { Zusammenfassung }}$}

Bei der Fest-Flüssig-Trennung feinstdisperser Suspensionen in Dekantierzentrifugen treten beim Transport des absedimentierten Feststoffs im konischen Teil der Maschine meist erhebliche Probleme auf. Der Schlamm läßt sich durch die Schnecke des Dekanters nicht oder nur bedingt fördern und so kommt es zu einem Feststoffrückstau, der sich bis in den zylindrischen Teil der Zentrifuge erstreckt und das Klärverhalten des Dekanters beeinträchtigt. Das Sediment kann entgegen seiner Förderrichtung sowohl durch den spalt zwischen Schneckenblatt und Konuswand als auch spiralig im Schneckenkanal zurückfließen.

Es wurde ein Rechenmodell entwickelt, mit dem es möglich ist, in Abhängigkeit von der Maschinengeometrie, der Betriebseinstellung und den rheologischen Eigenschaften des Sediments, Aussagen über die Förderfähigkeit bzw. über den möglichen Rückstau eines pastösen Stoffes in einer Dekantierzentrifuge zu treffen.

*) Vortrag auf dem Jahrestreffen 1985 der VerfahrensIngenieure in Hamburg, 25. bis 27. September 1985

**) Dipl.-Ing. Andreas Karolis (Vortragender) und Prof. Dr.-Ing. Werner Stahl, Institut für Mechanische Verfahrenstechnik und Mechanik der Universität Karlsruhe (TH), Kaiserstr. 12, D-7500 Karlsruhe 1 
Durch die Einführung der Begriffe Nutzungsgrad und Füllgrad kann das Betriebsverhalten des Dekanters besser beurteilt werden. Stellt man den Nutzungsgrad als Funktion der Schleuderziffer und der Differenzdrehzahl bzw. des Feststoffmassenzulaufs dar, so ist leicht zu ersehen, bei welchen Betriebseinstellungen die Maschine im Hinblick auf das Klärverhalten versagen bzw. noch störungsfrei funktionieren kann.

Ferner können Hinweise gegeben werden, wie die geometrischen Daten bzw. die Betriebsparameter $z u$ wählen sind, um Ergebnisse aus Pilotversuchen auf Großmaschinen übertragen zu können.

\section{Einleitung}

Bei der Verarbeitung von Suspensionen mit sehr feinen Partikelverteilungen $\left(x_{50}<10 \mu \mathrm{m}\right)$ in Dekantierzentrifugen treten neben den Grenzen durch die Klärwirkung besondere Probleme auch auf der Feststoffseite auf.

während leicht zu filtrierende und leicht $z u$ entwässernde körnige Feststoffe im allgemeinen problemlos von der

Schnecke aus dem zylindrischen über den konischen Teil des Rotors gefördert und ausgetragen werden können (Abb. 1), kommt es bei sedimenten mit pastöser, schlammartiger Konsistenz zu einem Rückstau.

Solche Stoffe widersetzen sich der Hochförderung durch die Schnecke, sie fließen konusabwärts in den zylindrischen

Teil des Dekanters zurück und beeinflussen damit die klärwirkung. Dieser Rückstau führt außerdem zu erhöhten Schnekkendrehmomenten und kann schließlich auch zu einem erhöhten Verschleiß der Schneckenblätter führen.

Für dieses Rückströmen der Paste im konischen Teil entgegen der Förderwirkung der Schnecke kommen zwei voneinander unabhängige Wege in Frage: 


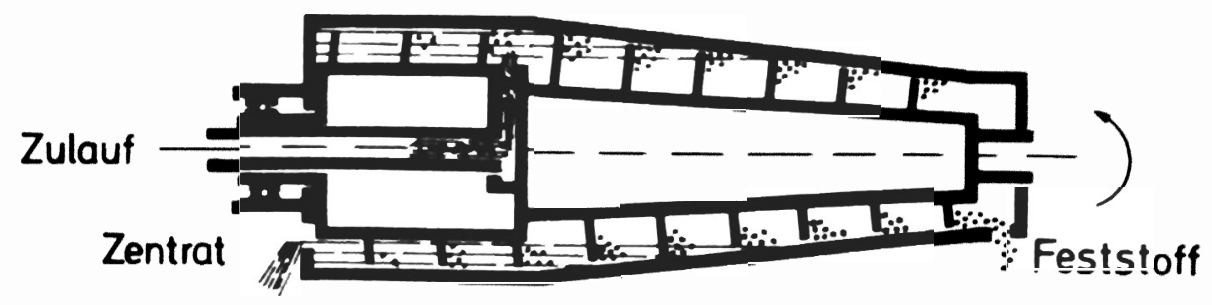

a)

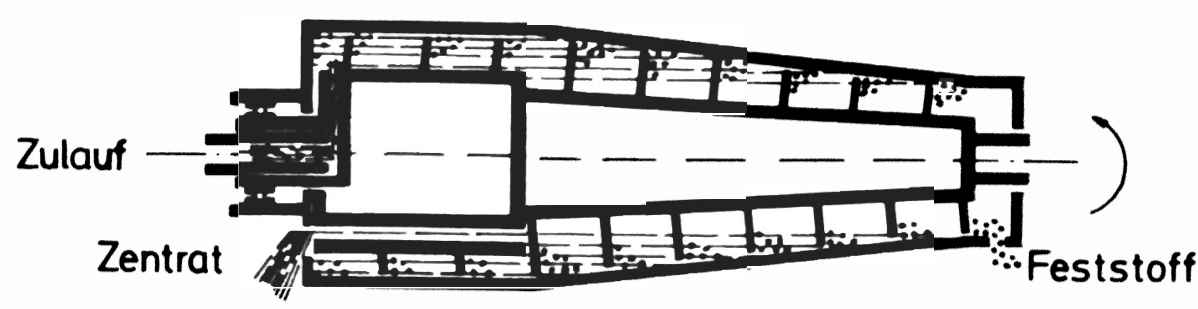

b)

Abb. 1: Funktionsprinzip einer Dekantierzentrifuge

a) Gegenstromprinzip, b) Gleichstromprinzip

a) Spaltrückströmung:

Die Paste fließt unter der Zentrifugalwirkung durch den Spalt zwischen der Schneckenblattspitze und der Konusinnenwand und sammelt sich an der Vorderseite des nächsttiefer gelegenen Kuchens an, der ebenso Material durch den nächsttiefer liegenden spalt weiter konusabwärts abgibt.

b) Spiralrückströmung:

Selbst bei völlig dichtem spalt wie er technisch aus Gründen der Sicherheit gegen metallische Berührung nicht hergestellt werden kann, verbleibt dem Schlamm noch ein Rückweg in den Sumpf. Die (gedachte) Verschneidungslinie zwischen dem Konus und den Schneckenblättern kann man sich über dem Umfang abgewickelt denken; damit wird klar, daß der einzelne Schneckenkanal ein Gefälle zum größeren Radius hin besitzt. Ebenso wie gedachterweise eine Kugel im Fliehkraftfeld in diesem Gerinne zum größeren Radius hin und in den zylinder hineinrollen würde, fließt der Schlamm, wenn seine Konsistenz nicht ausreichend steif ist, ebenfalls in diesem Gerinne bergab. 
Jeder Effekt einzeln - in Wirklichkeit aber meist die úberlagerung beider Effekte - hat zur Folge, daß der Vorwärtsförderung der Schnecke ein Rückstrom überlagert ist, der dazu führt, daß die Kuchenhöhe gegenüber der kontinuierlichen Förderung erheblich höher ansteigt.

Die Skizze (Abb. 2) zeigt schematisch vereinfacht einen solchen Rückstau, der stufenförmig von Gang zu Gang bis weit in den zylindrischen Teil hineinragt und die Klärung erheblich stört.

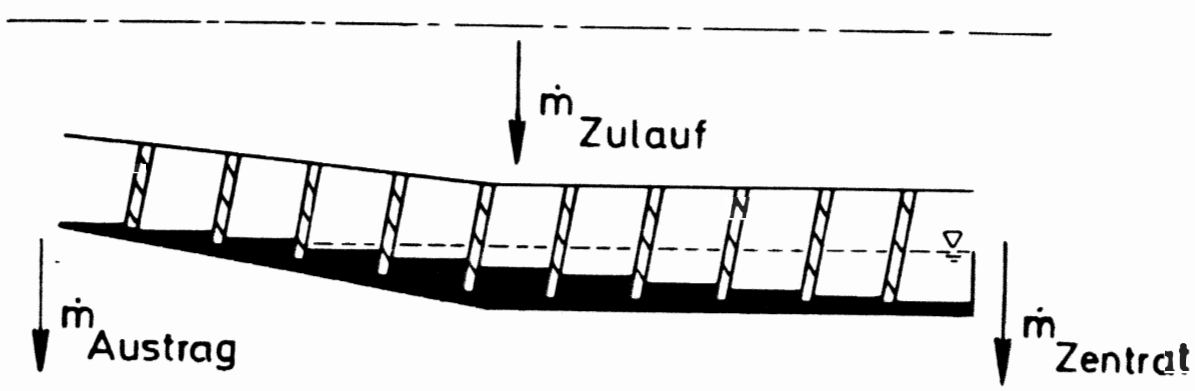

Abb. 2: Schematische Darstellung des Rückstaus im Dekanter

Die Existenz dieses Rückstaus während des Betriebszustandes ist vielfach bewiesen worden. Abb. 3 (Anhang) zeigt die Verstopfung einer Dekanterschnecke nach dem Ausbau. Während des kontinuierlichen Betriebs wurde der Ujberlastschutz ausgerückt und der suspensionszulauf gleichzeitig geschlossen $|1|$.

Obwohl der Dekanter über den Motor schnell elektrisch abgebremst wurde, kann die zurückgestaute Masse nicht in der Form ihres Gleichgewichtszustands erhalten werden; während des Auslaufens des Dekanters fließt der Schlamm durch die Spalte bzw. in Spiralrichtung weiter "hangabwärts" und man findet ihn radial weiter außen am konus abgelagert bzw. weiter in den zylinderteil der Trommel zurückgedrückt, als es dem Gleichgewichtszustand mit laufender schnecke und Feststoffdurchsatz entsprechen würde. 
Während die Transportvorgänge bei grobkörnigen gut entwässernden Produkten zum größten Teil durch eigene noch unveröffentlichte Arbeiten untersucht wurden, existiert bis heute keine Methode, um die "Förderfähigkeit" von Schlämmen unterschiedlicher Fließeigenschaften auf Maschinen verschiedener Geometrie oder Betriebseinstellung vorherzusagen. Man begnügt sich allein mit einer empirischen Vorgehensweise, wobei kaum Schlüsse auf andere Anwendungsfälle bezüglich Maschine und Produkt daraus gezogen werden können. So kann es z.B. vorkommen, daß die Funktionsweise eines Dekanters, der geometrisch ähnlich vergrößert wird, ganz zum Erliegen kommt (kein Feststoffaustrag), obwohl die Zentrifuge im Pilotmaßstab einwandfrei funktioniert hat.

2. Mathematische Erfassung des Transportvorgangs pastöser Stoffe im Dekanter

Im folgenden wird ein Rechenmodell vorgestellt, mit dem es möglich sein soll, die Transportvorgänge bzw. den Schlammrückstau im Dekanter in Abhängigkeit von der Maschinengeometrie, der Betriebseinstellung und den rheologischen Eigenschaften des sediments quantitativ zu erfassen bzw. zuverlässige Aussagen in Bezug auf "scale-up" treffen zu können.

\subsection{Die Feststoffmassenbilanz}

Die gesamte Feststoffmassenbilanz (Abb. 2) für einen Dekanter lautet:

$\dot{\mathrm{m}}_{\text {Zulauf }}=\dot{\mathrm{m}}_{\text {Austrag }}+\dot{\mathrm{m}}_{\text {Zentrat }}$ 

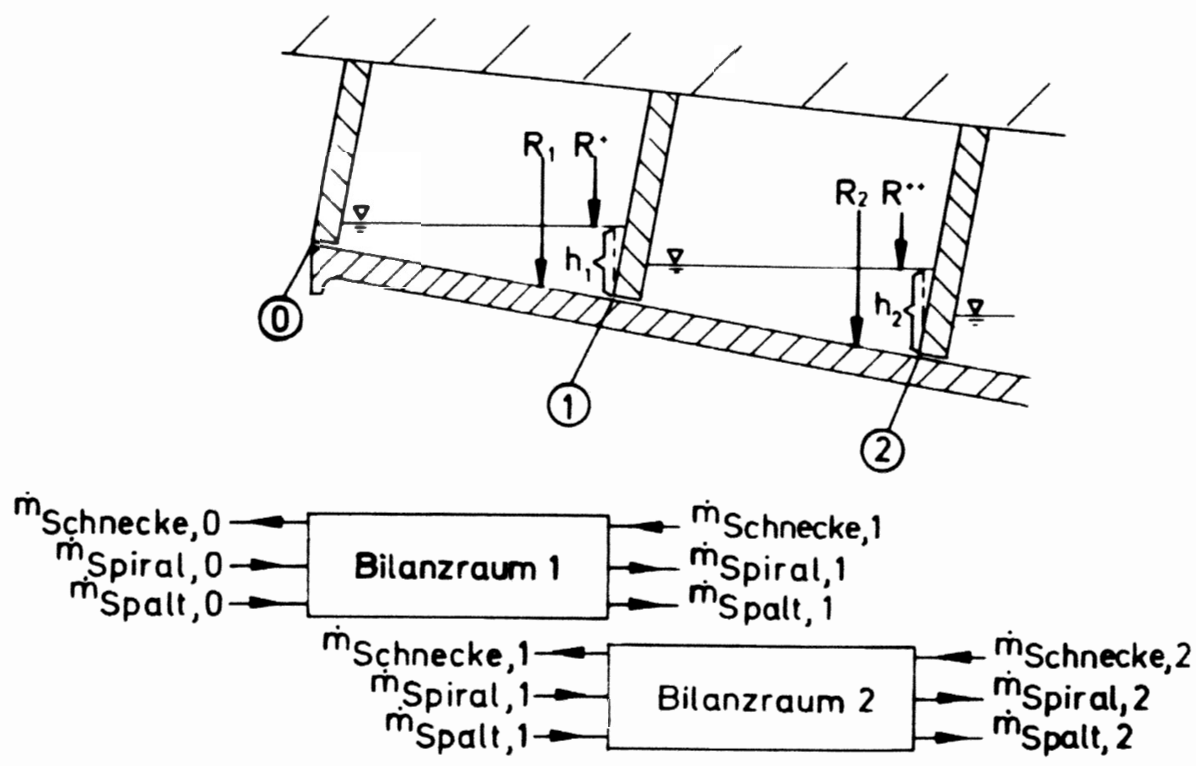

$\mathrm{Abb}$. 4: Teilmassenbilanz im Dekanter

Die Teilmassenbilanz für eine eingängige Schnecke lautet:

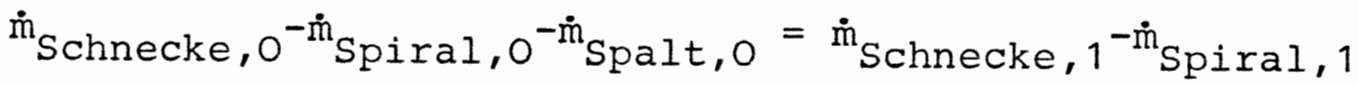

$$
-\dot{\mathrm{m}}_{\text {Spalt }, 1}=\dot{\mathrm{m}}_{\text {Schnecke, }} \mathrm{n}^{-\dot{\mathrm{m}}_{\text {Spiral }, \mathrm{n}}}{ }^{-\dot{\mathrm{m}}_{\text {Spalt }, \mathrm{n}}}
$$

Die folgenden Überlegungen gelten nun für den in der Praxis wichtigen Fall, daß die Spaltrückströmung $\dot{m}_{\text {Spalt, } n}=0$ ist (feste Grundschicht). Demnach lautet die Teilmassenbilanz:

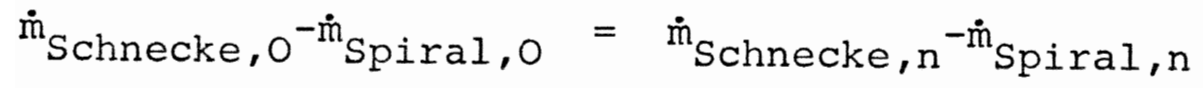

Unter der Annahme, daß unmittelbar am Austrag $\dot{\mathrm{m}}_{\text {Spiral, } 0}=0$ ist und mit $\dot{\mathrm{m}}_{\text {Schnecke, } \mathrm{O}}=\dot{\mathrm{m}}_{\text {Austrag }}$ vereinfacht sich $\mathrm{Gl}$. (3) $\mathrm{zu}$

$\dot{\mathrm{m}}_{\text {Austrag }}=\dot{\mathrm{m}}_{\text {Schnecke, } \mathrm{n}}{ }^{-\dot{\mathrm{m}}_{\text {Spiral }, \mathrm{n}}}$ 
Setzt man nun Gl. (4) in Gl. (1) ein, so folgt

$\dot{\mathrm{m}}_{\text {Zulauf }}=\dot{\mathrm{m}}_{\text {eff }, \mathrm{n}}+\dot{\mathrm{m}}_{\text {Zentrat }}$

wobei

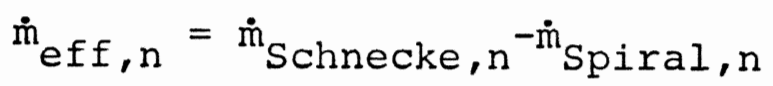

ist.

$\underline{2.2 \text { Berechnung der spiraligen Rückströmung }}$

Zur Berechnung der spiraligen Rückströmung werden folgende Annahmen gemacht:

- Die Strömung ist a) stationär

b) laminar

- Das Produkt ist inkompressibel und homogen

- Die Seitenwandeinflüsse sind vernachlässigbar ( $h<6-b$ )

- Ebenes, zweidimensionales Problem ( $h<R$ )

- Das Sediment hat freie, zylindrische oberfläche (keine Störung durch den Schneckengrundkörper)

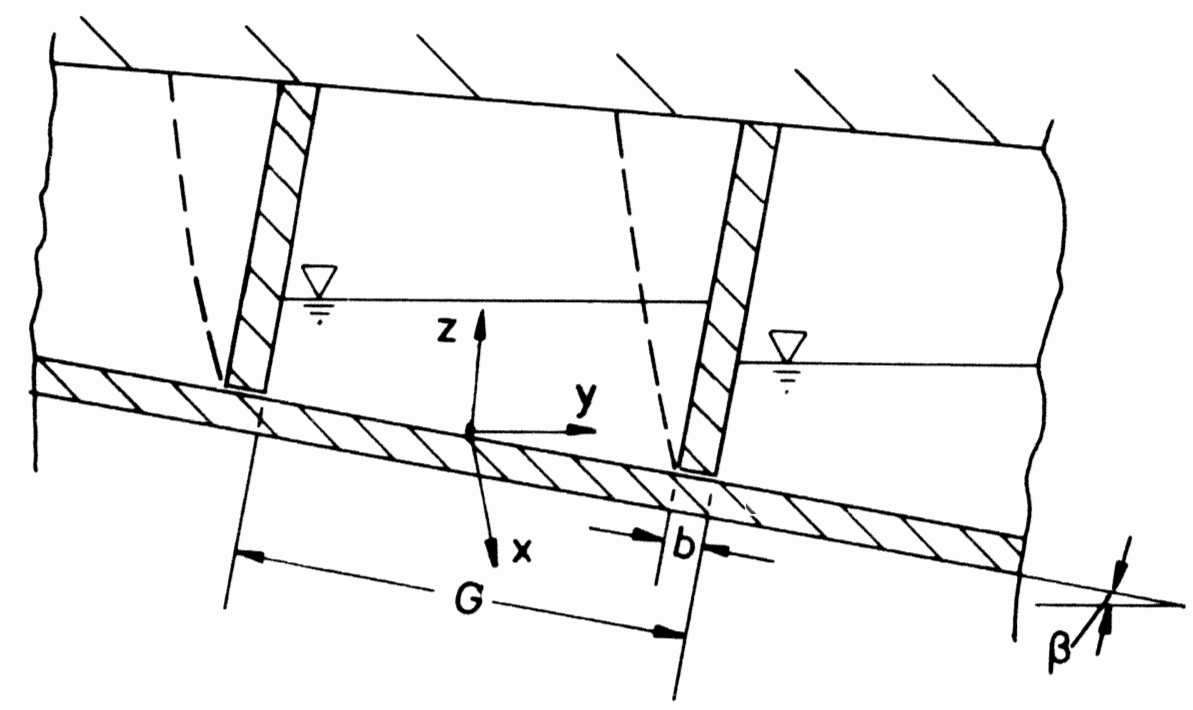

Abb. 5: Darstellung der Strömungsverhältnisse im Schneckenkanal 
Nun greift man aus dem Schneckenkanal ein Volumenelement $\mathrm{dV}$ (Abb. 5) heraus, und wendet man darauf die Impulsbilanz für die $x$ - und die $y$-Richtung an, so folgt:

$\tau_{z x}=\left(\frac{\partial p}{\partial x}-\rho \cdot g_{x}\right) \cdot\left(z-\frac{h}{\cos \delta}\right)$

für die $x$-Richtung, und

$\tau_{z y}=\left(\frac{\partial p}{\partial y}-\rho \cdot g_{y}\right) \cdot\left(z-\frac{h}{\cos \delta}\right)$

für die $y$-Richtung.

$\delta$ ist der sog. Gerinnewinkel oder auch Verschneidungswinkel, der sich aus dem Schneckensteigungswinkel $\alpha$ und dem Konuswinkel $\beta$ berechnen läßt:

$\delta=\operatorname{arc} \sin (\sin \alpha \cdot \sin \beta)$

mit

$\alpha=\arctan \left(\frac{G}{2 \pi \cdot R}\right)$

Das Fließen solcher hochkonzentrierter Suspensionen bzw. Sedimente kann je nach Wandrauhigkeit große Unterschiede aufweisen. D.h. die spiralige Rückströmung kann nicht allein durch die Scherfunktion des Mediums beschrieben werden, wo Wandhaftung angenommen wird (vor allem dann, wenn keine feste Grundschicht vorhanden ist), sondern muB auch die Gleitfunktion des Materials einbezogen werden, damit auch der Einfluß der Oberflächenrauhigkeit der wand auf die spiralige Rückströmung berücksichtigt wird $|2|$.

2.2.1 Berechnung des Schervolumenstroms $\dot{\mathrm{V}}_{\text {scher }}$

Zur Berechnung des Schervolumenstroms muß die Scherfunktion eingeführt werden, die in allgemeiner Darstellung

$\tau=\tau_{0}+K \cdot \dot{\gamma}^{n}$ 
lautet (Abb. 6).

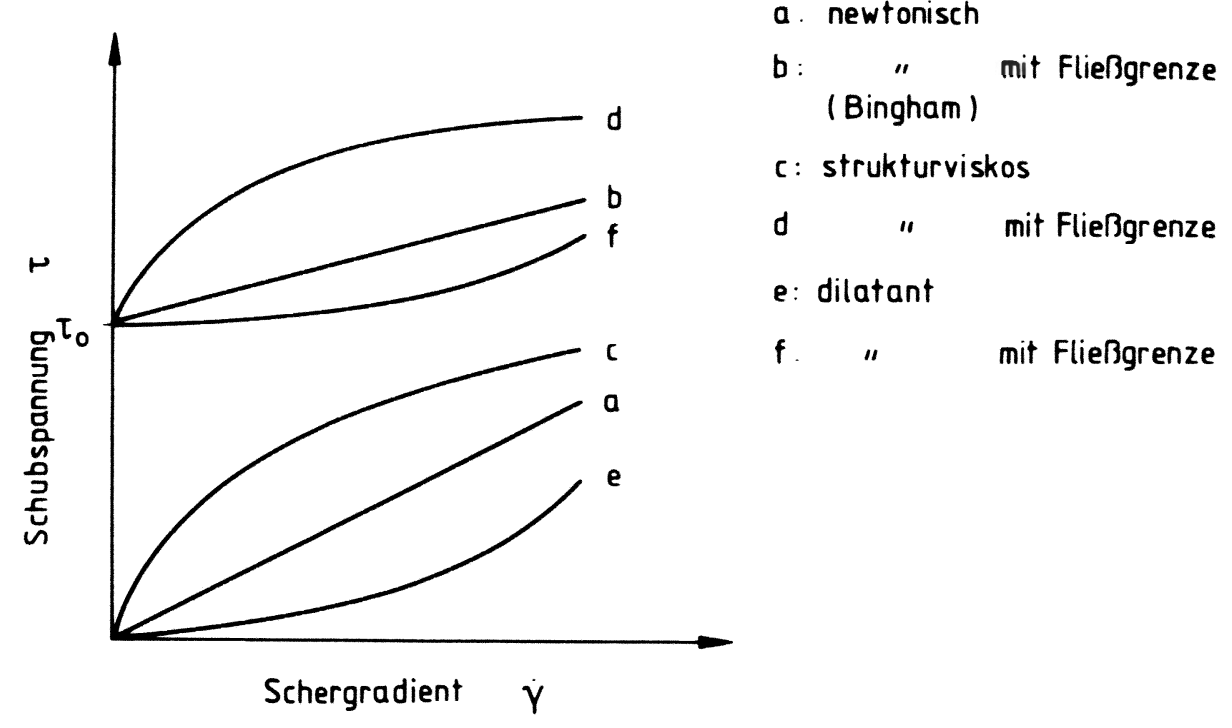

Abb. 6: Qualitative Darstellung von Fließfunktionen verschiedener Medien

Wenn man annimmt, daß der Schergradient $\dot{\gamma}_{x}$ entlang des Schneckenkanals ( $x$-Richtung) viel größer ist als der Schergradient $\dot{\gamma}_{y}$ in $y$-Richtung, was für flache Schneckensteigungen auch zutrifft, geht Gl. (11) in

$\tau_{z x}=\tau_{O}+K \cdot \dot{\gamma}_{x}^{n}$

über.

Setzt man nun Gl. (12) in Gl. (7) ein, so folgt für den schergradienten $\dot{\gamma}_{\mathrm{x}}$ :

$\dot{\gamma}_{x}=\frac{d v_{x}}{d z}=\left[\frac{\left(\frac{\partial p}{\partial x}-\rho \cdot g_{x}\right) \cdot\left(z-\frac{h}{\cos \delta}\right)-\tau_{0}}{k}\right]^{1 / n}$ 
Durch Integration von Gl. (13) folgt:

$$
\begin{gathered}
v_{x}=\frac{n}{n+1} \cdot \frac{1}{\left(\frac{\partial p}{\partial x}-\rho \cdot g_{x}\right)} \cdot k^{-\frac{1}{n}} \cdot\left[\left(\frac{\partial p}{\partial x}-\rho \cdot g_{x}\right)\right. \\
\left.\cdot\left(z-\frac{h}{\cos \delta}\right)-\tau_{0}\right]^{\frac{n+1}{n}}+c_{1}
\end{gathered}
$$

Da hochkonzentrierte Suspensionen eine ausgeprägte Fließgrenze $\tau_{0}$ aufweisen, welche überschritten werden muß, damit das Material zu fließen beginnt, muß man an dieser stelle zwischen einem gescherten Bereich und einem ungescherten Bereich unterscheiden, wo das Medium propfenförmig fließt $(\mathrm{Abb}, 7)$ :

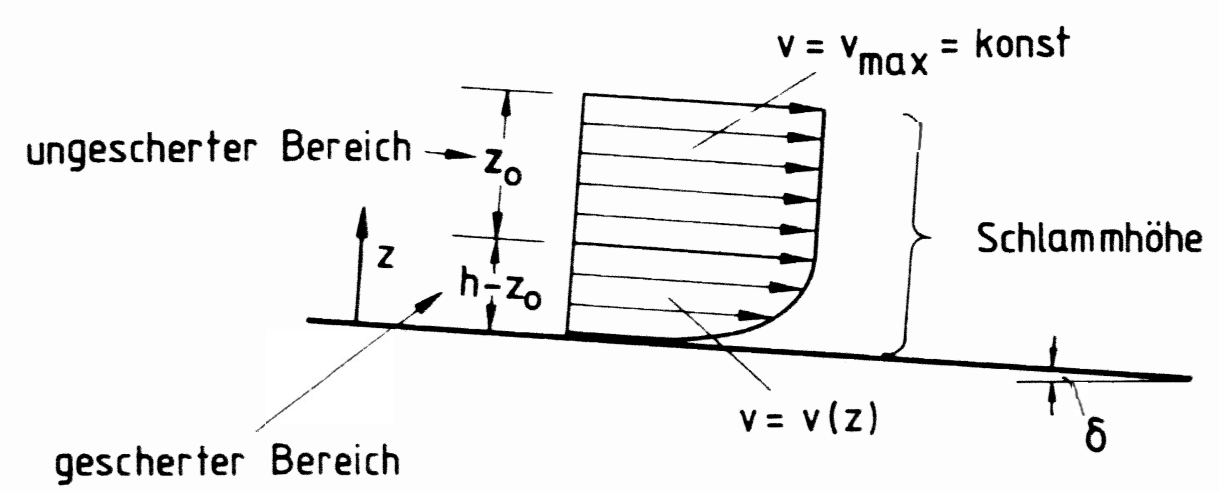

Abb. 7: Geschwindigkeitsprofil für die Scherströmung mit Fließgrenze $\tau_{0}$

a) Gescherter Bereich: $0 \leq z \leq \frac{h-z_{0}}{\cos \delta}$

Mit der Randbedingung $\mathrm{v}_{\mathrm{x}}(\mathrm{z}=0)=0$ (Wandhaftung vorausgesetzt) lautet Gl. (14) für den gescherten Bereich: 


$$
\begin{aligned}
v_{x} & =\frac{n}{n+1} \cdot k^{-1 / n} \cdot\left(\frac{\partial p}{\partial x}-\rho \cdot g_{x}\right)^{1 / n} \cdot \\
& \left\{\left[z-\frac{h}{\cos \delta}-\frac{\tau_{O}}{\left(\frac{\partial p}{\partial x}-\rho \cdot g_{x}\right)}\right]^{\frac{n+1}{n}}-\left[-\frac{h}{\cos \delta}-\frac{\tau_{0}}{\left(\frac{\partial p}{\partial x}-\rho \cdot g_{x}\right)}\right]^{\frac{n+1}{n}}\right\}
\end{aligned}
$$

b) Ungescherter Bereich (Propfströmung): $\frac{h-z_{0}}{\cos \delta} \leq z \leq \frac{h}{\cos \delta}$ Mit der Randbedingung $\frac{d v_{x}}{d z}\left(z=\frac{h-z_{0}}{\cos \delta}\right)=0$ folgt aus GI.

der Höhenanteil $z_{0}$ des ungescherten Bereiches:

$z_{0}=\frac{-\tau_{0} \cdot \cos \delta}{\left(\frac{\partial p}{\partial x}-\rho \cdot g_{x}\right)}$

und aus Gl. (15) folgt schließlich:

$$
\begin{gathered}
v_{x}=v_{\max }=-\frac{n}{n+1} \cdot k^{-1 / n} \cdot\left(\frac{\partial p}{\partial x}-\rho \cdot g_{x}\right)^{1 / n} . \\
\cdot\left[-\frac{h}{\cos \delta}-\frac{\tau_{0}}{\left(\frac{\partial p}{\partial x}-\rho \cdot g_{x}\right)}\right]^{\frac{n+1}{n}}
\end{gathered}
$$

Der Schervolumenstrom im Schneckenkanal ergibt sich dann durch erneute Integration von $\mathrm{v}_{\mathrm{x}}$ und $\mathrm{v}_{\max }$ :

$$
\dot{\mathrm{V}}_{\text {scher }}=(\mathrm{G}-\mathrm{b}) \cdot \cos \alpha \cdot\left[\int_{0}^{\frac{\mathrm{h}-\mathrm{z}_{0}}{\cos \delta}} \mathrm{v}_{\mathrm{x}} \mathrm{dz}+\int_{\frac{\mathrm{h}-\mathrm{z}_{0}}{\cos \delta}}^{\frac{\mathrm{h}}{\cos \delta}} \mathrm{v}_{\max } \mathrm{dz}\right]
$$

bzw.

$$
\begin{aligned}
\dot{V}_{\text {scher }} & =(G-b) \cdot \cos \alpha \cdot \frac{n}{n+1} \cdot K^{-1 / n} \cdot\left(\frac{\partial p}{\partial x}-\rho \cdot g_{x}\right)^{1 / n} \cdot\left(-\frac{\left(h-z_{O}\right)}{\cos \delta}\right) \frac{2 n+1}{n} . \\
& {\left[\left(\frac{n+1}{2 n+1}\right)+\left(\frac{z_{O}}{h-z_{O}}\right)\right] }
\end{aligned}
$$


Ersetzt man die Druckänderung durch die Stauhöhenänderung des zurückfließenden Mediums, so ergibt sich:

$$
\left(\frac{\partial p}{\partial x}-\rho \cdot g_{x}\right)=4 \pi^{2} \cdot n_{H}^{2} \cdot \bar{\rho} \cdot R \cdot \sin \alpha \cdot \cos \beta \cdot\left(\frac{\partial h}{\partial I}-\tan \beta\right)
$$

Hierin ist 1 die Ortskoordinate parallel zur Drehachse der Zentrifuge. Für den Fall, daß das sediment unter dem Flüssigkeitsniveau sich befindet, mu in Gl. (20) $\bar{\rho}$ durch $\left(\bar{\rho}-\rho_{1}\right)$ ersetzt werden. Mit Gl. (20) nimmt Gl. (19) schließlich folgende Form an:

$$
\begin{aligned}
\dot{\mathrm{V}}_{\text {scher }}= & (G-b) \cdot \cos \alpha \cdot \frac{\mathrm{n}}{\mathrm{n}+1} \cdot \mathrm{K}^{-1 / n} \cdot\left(4 \pi^{2} \cdot \bar{\rho} \cdot R \cdot \mathrm{n}_{\mathrm{H}}^{2} \cdot \sin \alpha \cdot \cos \beta\right)^{1 / n} . \\
& \left(\frac{\mathrm{dh}}{\mathrm{dl}}-\tan \beta\right)^{1 / n} \cdot\left(-\frac{\left(\mathrm{h}-\mathrm{z}_{\mathrm{O}}\right)}{\cos \delta}\right)^{\frac{2 \mathrm{n}+1}{\mathrm{n}}} \cdot\left[\left(\frac{\mathrm{n}+1}{2 \mathrm{n}+1}\right)+\frac{\mathrm{z}_{O}}{\mathrm{~h}-\mathrm{z}_{\mathrm{O}}}\right]
\end{aligned}
$$

wobei

$$
z_{0}=\frac{-\tau_{0} \cdot \cos \delta}{4 \pi^{2} \cdot n_{H}^{2} \cdot \bar{\rho} \cdot R \cdot \sin \alpha \cdot \cos \beta \cdot\left(\frac{d h}{d I}-\tan \beta\right)}
$$

ist. Setzt man nun GI. (16a) in GI. (21) ein, so ergibt sich für den Schervolumenstrom $\dot{\mathrm{V}}_{\text {scher }}$

$$
\begin{aligned}
\dot{V}_{\text {scher }} & =\frac{n}{n+1} \cdot K^{-1 / n} \cdot(G-b) \cdot \cos \alpha \cdot \frac{h}{A} \cdot\left(h \cdot A-\tau_{0}\right)^{\frac{n+1}{n}}\left(\frac{n+1}{2 n+1}+\frac{n}{2 n+1} .\right. \\
& \left.\cdot \frac{\tau_{O}}{h \cdot A}\right)
\end{aligned}
$$

mit

$A=4 \pi^{2} \cdot n_{H}^{2} \cdot \bar{\rho} \cdot R \cdot \sin \alpha \cdot \cos \beta \cdot\left(\tan \beta-\frac{d h}{d l}\right)$ 
2.2.2 Berechnung des Gleitvolumenstroms $\dot{\mathrm{V}}_{\text {Gleit }}$

Zur Berechnung des Gleitvolumenstroms wird die Gleitfunktion eingeführt (Abb. 8):

$$
\tau_{z x}=C^{\prime} \cdot v_{g_{x}}^{m}+\tau_{1}
$$

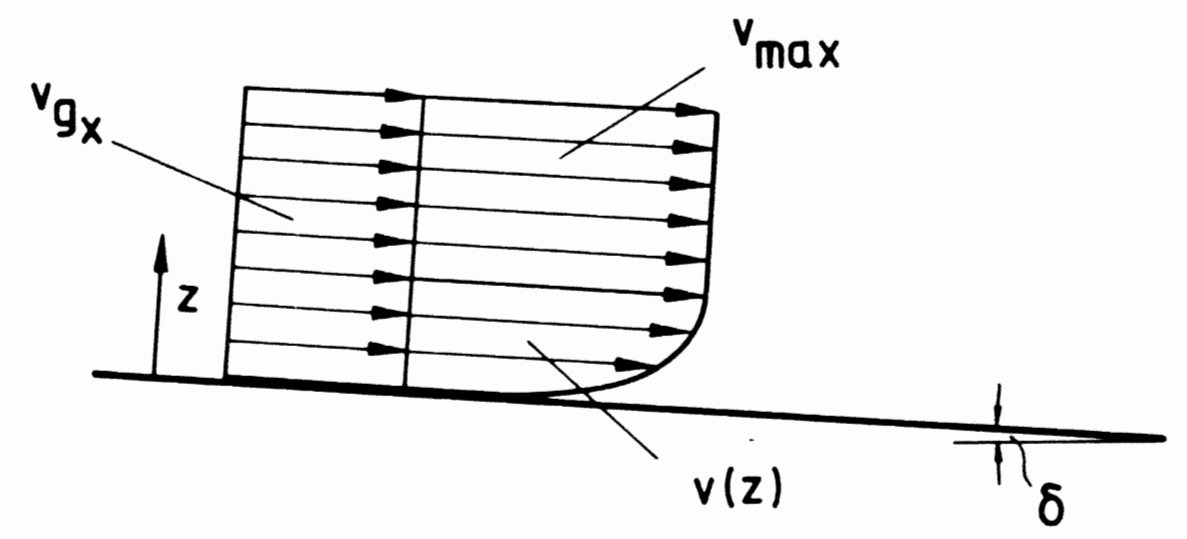

Abb. 8: Geschwindigkeitsprofil für Gleit-Scherströmung

Mit der Randbedingung $\tau_{z x}(z=0)=\tau_{W}$ folgt aus Gl. (23) und Gl. (7) für die Gleitgeschwindigkeit $v_{g_{X}}$

$v_{g_{x}}=\left[\frac{\left(\frac{\partial p}{\partial x}-\rho \cdot g_{x}\right) \cdot\left(-\frac{h}{\cos \delta}\right)-\tau_{1}}{C^{\prime}}\right]^{1 / m}$

Der Gleitvolumenstrom ergibt sich dann $z u$

$$
\dot{V}_{\text {Gleit }}=(G-b) \cdot \cos \alpha \cdot h \cdot\left[\frac{\left(\frac{\partial p}{\partial x}-\rho \cdot g_{x}\right) \cdot\left(-\frac{h}{\cos \delta}\right)-\tau_{1}}{C^{\prime}}\right]^{1 / m}
$$


bzw.

$$
\begin{aligned}
\dot{\mathrm{V}}_{\text {Gleit }} & =(\mathrm{G}-\mathrm{b}) \cdot \cos \alpha \cdot \mathrm{h} \cdot \\
& \cdot\left[\frac{4 \pi^{2} \cdot \mathrm{n}_{\mathrm{H}}^{2} \cdot \bar{\rho} \cdot \mathrm{R} \cdot \sin \alpha \cdot \cos \beta \cdot\left(\frac{\mathrm{dh}}{\mathrm{dl}}-\tan \beta\right)\left(-\frac{\mathrm{h}}{\cos \delta}\right)-\tau_{1}}{\mathrm{C}^{\prime}}\right]_{(26)}^{1 / \mathrm{m}}
\end{aligned}
$$

Der spiralige Feststoffmassenstrom $\dot{m}_{\text {Spiral }}$ läßt sich dann wie folgt berechnen:

$\dot{\mathrm{m}}_{\text {Spiral }}=\bar{\rho} \cdot(1-\mathrm{RF}) \cdot \dot{\mathrm{V}}_{\text {Spiral }}$

mit

$\dot{\mathrm{V}}_{\text {Spiral }}=\dot{\mathrm{V}}_{\text {Scher }}+\dot{\mathrm{V}}_{\text {Gleit }}$

2.2.3 Berechnung der Schneckenförderung $\dot{m}_{S c l}$

Schnecke

Zur Berechnung der effektiven Förderung $\dot{m}_{\text {eff }}$ muß noch der Anteil der von der Schnecke gefördert wird, berücksichtigt werden. Dies ergibt sich über die Differenzbewegung zwischen Schneckenkörper und Trommel zu

$\dot{\mathrm{m}}_{\text {Schnecke }}=\bar{\rho} \cdot(1-\mathrm{RF}) \cdot 2 \pi \cdot \mathrm{R} \cdot \mathrm{h} \cdot(\mathrm{G}-\mathrm{b}) \cdot \mathrm{n}_{\mathrm{Diff}} \cdot \cos ^{2} \alpha$

2.3 Gesamte Transportgleichung $\left(\dot{\mathrm{m}}_{\text {Spalt }}=0\right)$

Setzt man nun Gl. (27) und Gl. (29) in Gl. (5) ein, so

folgt für die gesamte Transportgleichung: 


$$
\begin{aligned}
& \dot{\mathrm{m}}_{\mathrm{Zulauf}}=\bar{\rho} \cdot(1-\mathrm{RF}) \cdot\left\{2 \pi \cdot \mathrm{R} \cdot \mathrm{h} \cdot(\mathrm{G}-\mathrm{b}) \cdot \mathrm{n}_{\mathrm{Diff}} \cdot \cos ^{2} \alpha-\right. \\
& (G-b) \cdot \cos \alpha \cdot \frac{n}{n+1} \cdot K^{-1 / n} \cdot\left(4 \pi^{2} \cdot \bar{\rho} \cdot R \cdot n_{H}^{2} \cdot \sin \alpha \cdot \cos \beta\right)^{1 / n} \cdot \\
& \left(\frac{\mathrm{dh}}{\mathrm{dl}}-\tan \beta\right)^{1 / \mathrm{n}} \cdot\left(-\left(\frac{\mathrm{h}-\mathrm{z}_{O}}{\cos \delta}\right)^{\frac{2 \mathrm{n}+1}{\mathrm{n}}} \cdot\left[\left(\frac{\mathrm{n}+1}{2 \mathrm{n}+1}\right)+\left(\frac{\mathrm{z}_{O}}{\mathrm{~h}-\mathrm{z}_{\mathrm{O}}}\right)\right]-\right. \\
& (G-b) \cdot \cos \alpha \cdot h \cdot C^{\prime-1 / m} \cdot\left[4 \pi^{2} \cdot \bar{\rho} \cdot R \cdot n_{H}^{2} \cdot \sin \alpha \cdot \cos \beta\right. \text {. } \\
& \left.\left.\cdot\left(\frac{d h}{d l}-\tan \beta\right) \cdot\left(-\frac{h}{\cos \delta}\right)-\tau_{1}\right]^{1 / m}\right\}+\dot{m}_{\text {Zentrat }}
\end{aligned}
$$

Der Anteil des Gleitvolumenstroms $\dot{\mathrm{V}}_{\text {Gleit }}$ in Gl. (30) wurde, wegen der Annahme einer festen Grundschicht, bei den folgenden Berechnungen nicht berücksichtigt. Er sei aber hier der Vollständigkeit halber aufgeführt. Für den Fall, daß die Paste auch durch den Spalt zwischen Schneckenblatt und Konusinnenwand zurückfließen kann (keine feste Grundschicht), muß nämlich bei der spiraligen Rückströmung $\dot{V}_{\text {Spiral }}$ der Anteil von $\dot{V}_{\text {Gleit }}$ auch berücksichtigt werden (abhängig von der Oberflächenrauhigkeit der Wand).

Bei Gl. (30) handelt es sich um eine gewöhnliche Differentialgleichung höherer ordnung, die analytisch nicht lösbar ist. Als unbekannte Größen treten dabei die örtliche stauhöhe $\mathrm{h}$ bzw. die änderung der Höhe $\mathrm{h}$ mit der $\ddot{n}$ derung des Ortes $1\left(\frac{d h}{d l}\right)$ auf. Zur Lösung der Gl. (30) muß deswegen numerisch vorgegangen werden.

\section{Numerische Berechnung des Rückstauprofils}

Zur Berechnung des Rückstauprofils wird - ausgehend vom Austragsradius als startpunkt - iterativ konusabwärts gerechnet. Um die Iteration zu starten, benötigt man eine starthöhe 'h ${ }_{\text {Start' }}$ die sich aus der Randbedingung $\dot{\mathrm{m}}_{\text {Spiral }}=0 \mathrm{am}$ Austragsradius zu 
$h_{\text {Start }}=\frac{\dot{\mathrm{m}}_{\text {Zulauf }}}{\bar{\rho} \cdot(1-R F) \cdot 2 \pi \cdot \mathrm{R}_{\text {Austrag }} \cdot(G-b) \cdot \mathrm{n}_{\text {Diff }} \cdot \cos ^{2} \alpha}$

ergibt.

Wie man aus Gl. (31) ersehen kann, würde die Starthöhe

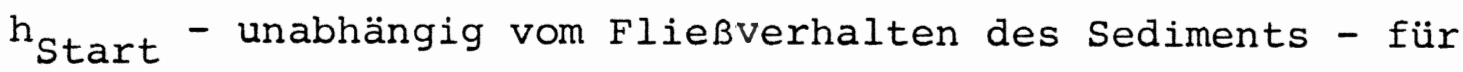

$\frac{\stackrel{\circ}{\text { Zulauf }}_{\text {Diff }}}{\mathrm{n}_{\text {if }}} \rightarrow \infty$

ins Unendliche anwachsen. Je nach Konsistenz wird aber der Schlamm aus der Zentrifuge ausgeworfen, sobald die Schlammoberfläche den Austragsradius erreicht hat (Abb. 9).

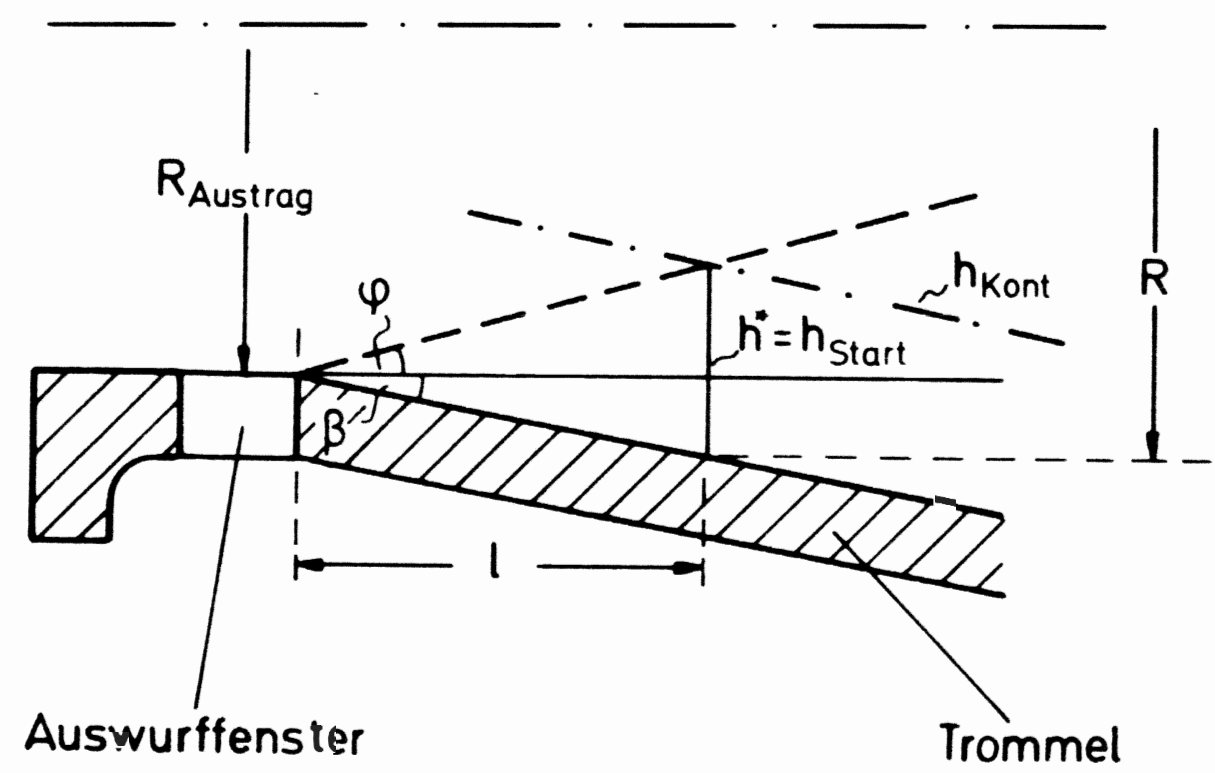

Abb. 9: Schematische Darstellung der Startbedingung

Die Starthöhe $h_{\text {Start }}$ bzw. der Startradius $R_{\text {Start }}$ ergibt sich dann aus dem schnittpunkt der kontinuierlichen Höhe $h_{\text {kont }}$ (d.h. wenn die Zentrifuge rückstaufrei arbeiten würde)

$\mathrm{h}_{\text {kont }}=\frac{\stackrel{\mathrm{m}}{\text { Zulauf }}}{\bar{\rho} \cdot(1-\mathrm{RF}) \cdot 2 \pi \cdot \mathrm{R} \cdot(\mathrm{G}-\mathrm{b}) \cdot \mathrm{n}_{\mathrm{Diff}} \cdot \cos ^{2} \alpha}$ 
und der Höhe $\mathrm{h}^{*}$, die sich nach Abb. 9 zu

$h^{*}=R-R_{\text {Austrag }}+\sqrt{\frac{3600 \cdot \tau_{O} \cdot\left(R-R_{\text {Austrag }}\right)}{4 \pi^{2} \cdot \bar{\rho} \cdot R \cdot n_{H}^{2} \cdot \tan \beta}}$

berechnen läßt ( $h_{\text {kont }}=h^{*}=h_{\text {Start' }}$ bei $\left.\mathrm{R}=\mathrm{R}_{\text {Start }}\right)$. Mit dem so ermittelten startwert geht man dann in die Gl. (30) und berechnet daraus $\frac{d h}{d l}$. Die stauhöhe für den nachfolgenden Iterationsschritt ergibt sich somit zu

$h_{m+1}=\left(I_{m+1}-I_{m}\right) \cdot \frac{d h}{d l}+h_{m}$

wobei $h_{m}$ beim ersten Schritt gleich $h_{\text {Start }}$ ist. Die so berechneten Rückstauhöhen ergeben schließlich das gesamte Rückstauprofil über den ganzen Dekanter.

$\mathrm{Abb}$. 10 zeigt ein berechnetes Rückstauprofil für eine Maschine bestimmter Geometrie und Betriebseinstellung.

(Der Start erfolgt am Austragsradius)

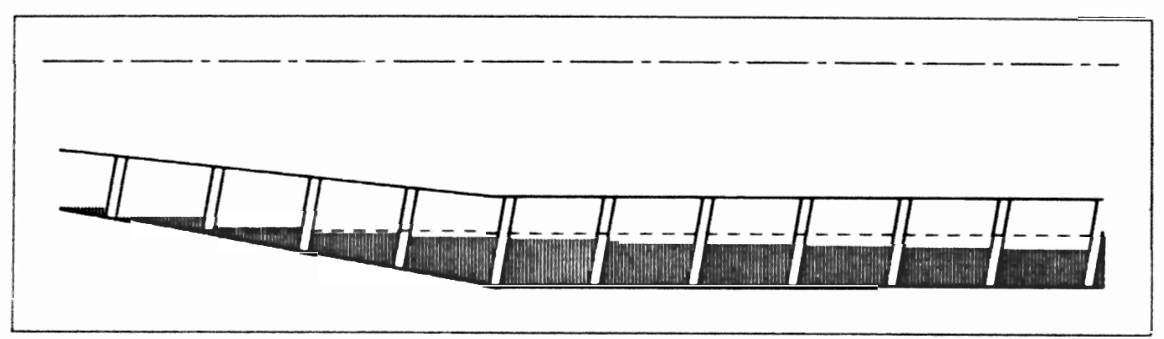

Maschinentyp: A

Zylinderdurchmesser $D_{2 y 1}=250 \mathrm{~mm}$ Austragsdurchmesser $D_{\text {Nustr }}=165 \mathrm{~mm}$ Niveaudurchmesser $\quad D_{\text {wiv }}=190 \mathrm{~mm}$ Zylinderlânge $\quad L_{2 y 1}=340 \mathrm{~mm}$ Konuswinke] $\quad B=10 \mathrm{Grad}$ Ganghőhe Blattstårke Niveauvolumen

\begin{tabular}{|c|c|c|}
\hline Rotordrehzahl & $n_{H}$ & $=3000 \mathrm{~g} / \mathrm{min}$ \\
\hline Differenzdrehzahl & $n_{\text {odff }}$ & $=30 \mathrm{1} / \mathrm{min}$ \\
\hline Festst of fdurchsatz & $m_{s}$ & $=300 \mathrm{~kg} / \mathrm{h}$ \\
\hline Restfeuchte & RF 1 & $=33.0 \mathrm{Gew}-\mathrm{x}$ \\
\hline ' & RF2 & $=39.2 \mathrm{Gew}-\mathrm{x}$ \\
\hline ' & AF3 & $=48.0 \mathrm{Gew}-\mathbf{x}$ \\
\hline Nutzungsgrad & NG & $=19.2 *$ \\
\hline Füllgrad & FG & $=4.6$ \\
\hline
\end{tabular}

Abb. 10: Berechnetes Rückstauprofil 
Es wurden dabei zwei Begriffe eingeführt, mit denen die Funktionsfähigkeit des Dekanters besser beurteilt werden kann:

a) Nutzungsgrad NG: Er ist ein Maß für das noch zur Verfügung stehende Klärvolumen und wird wie folgt definiert:

$N G=\left(1-\frac{V S 1}{V N I V}\right) \cdot 100$ in $\%$

wobei VNIV das Niveauvolumen und VS1 das Schlammvolumen ist, welches das Niveauvolumen verdrängt.

b) Füllgrad FG: Damit kann beurteilt werden, wieviel Schlamm mehr sich in dem Dekanter ansammelt, als wenn dieser rückstaufrei arbeiten würde. Er ist ein Maß für die tatsächliche Verweilzeit des Sediments in der Maschine und ist definiert als das Verhältnis aus dem gesamten Schlammvolumen durch Rückstau (VS) bezogen auf das gesamte Schlammvolumen ohne Rückstau (VK):

$$
F G=\frac{V S}{V K}
$$

Bei der Berechnung solcher Rückstauprofile besteht die größte Schwierigkeit darin, daß die örtliche Restfeuchte des sediments nicht bekannt ist. Die stoffdaten $\left(\tau_{0}, k, n_{1} \tau_{1}\right.$, $C, m)$ sind jedoch von der Restfeuchte abhängig. Um dies zu berücksichtigen, wurde folgendermaßen vorgegangen: Es wurden die Restfeuchten RF1, RF2 und RF3 eingeführt, wobei RF1 die Restfeuchte am Feststoffaustrag ist, RF2 jene am Übergang Konus-zylinder und RF3 die Restfeuchte am Zylinderende, wo die Suspension im Falle eines Gleichstromdekanters aufgegeben wird.

Die Austragsrestfeuchte RF1 kann gemessen werden. Trägt man diese Restfeuchte über einer als "Pressung" definierten Größe

$P=\frac{m_{s} \cdot C}{n_{D i f f}}$ 
in doppellogarithmischem Maßstab auf, so ergibt sich ein abfallender Kurvenverlauf mit gleichbleibender steigung, der bei sehr großen P-Werten in eine Horizontale einmündet (Abb. 11).

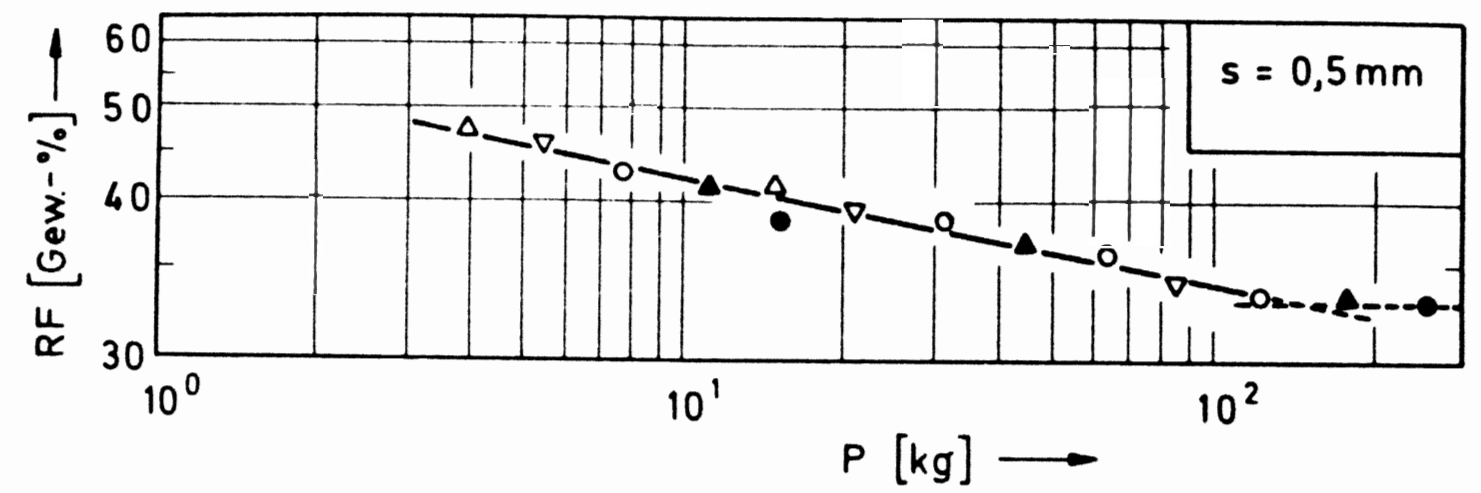

Abb. 11: EinfluB der Pressung auf die Austragsrestfeuchte

Da die Spaltweite bei diesem Versuch klein war ( $s=0,5 \mathrm{~mm}$ ), konnte angenommen werden, daß der Volumenrückstrom durch den Spalt vernachlässigbar gering ist (feste Grundschicht). Während im größten Bereich des Diagramms eine Abhängigkeit der Restfeuchte mit steigender Pressung vorliegt, scheint die Kompressibilität des Sediments am Knickpunkt der Kurve plötzlich erschöpft $z u$ sein. Durch einen relativ geringen Versuchsaufwand ( $3 \mathrm{bzw}$. 4 Meßpunkte) kann somit die Aus'tragsrestfeuchte RF1 für verschiedene Betriebszustände ( $\dot{m}_{S}$ ' $\mathrm{C}, \mathrm{n}_{\mathrm{Diff}}$ ) bestimmt werden und die Funktion $\mathrm{RF} 1=\mathrm{f}(\mathrm{P})$ aufgestellt werden.

Die Restfeuchte RF3 wurde mit 48 Gew. $\%$ festgesetzt (gemessen am ausgebauten Dekanter) und für alle Betriebszustände in erster Näherung als konstant angenommen. 
Zur Ermittlung der Änderung der Restfeuchte über der ganzen Maschinenlänge und damit auch des Wertes für RF2 wurde ein linearer Restfeuchteverlauf zwischen RF1 und RF3 angenommen. In dem oben gezeigten Beispiel zur Berechnung des Rückstauprofils (Abb. 10) kann man ersehen, daß nur 19,2 \% des gesamten Niveauvolumens für die Klärung zur Verfügung stehen. Für dieses Beispiel würde sich der Feststoff um das 4,6 fache länger in der zentrifuge aufhalten, als wenn die Maschine rückstaufrei funktionieren würde. Setzt man nun die Rotordrehzahl von $3000 \mathrm{~min}^{-1}$ auf $2750 \mathrm{~min}^{-1}$ herab (Abb. 12), so kann man sehen, daß der Nutzungsgrad von $19,2 \%$ auf $73,4 \%$ ansteigt.

RÜCKSTAUPROF ILBERECHNUNG

(Der Start erfolgt am Austragsradius)

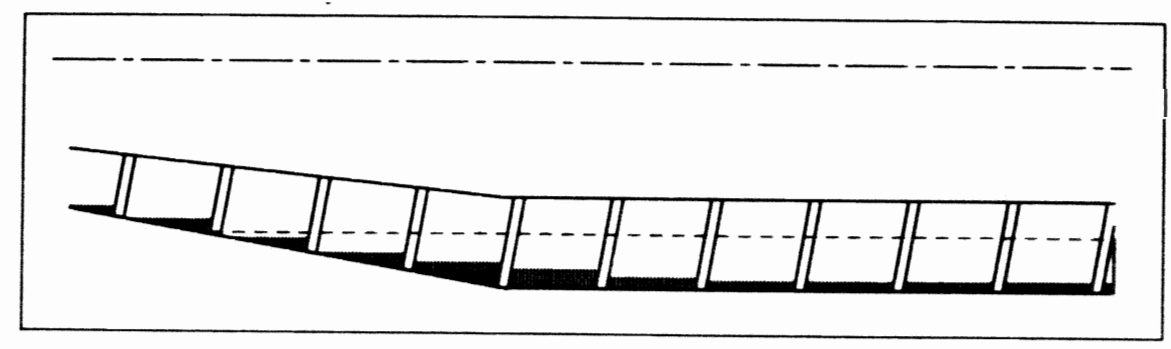

Maschinentyp : A

$\begin{array}{ll}\text { Zylinderdurchmesser } & D_{2 y 1}=250 \mathrm{~mm} \\ \text { Austragsdurchmesser } & D_{\text {wustr }}=165 \mathrm{~mm} \\ \text { Niveaudurchmesser } & D_{\text {wiv }}=190 \mathrm{~mm} \\ \text { Zylinderlănge } & L_{2 y 1}=340 \mathrm{~mm} \\ \text { Konuswinkel } & B=10 \mathrm{Grad} \\ \text { Ganghöhe } & G=55 \mathrm{~mm} \\ \text { Blattstărke } & D=6 \mathrm{~mm} \\ \text { Niveauvolumen } & V_{\text {wiv }}=7.8 \mathrm{dm}^{3}\end{array}$

Produkt : KAOLIN

\begin{tabular}{|c|c|c|}
\hline Rotordrehzahl & $n_{H}$ & $=27501 / \mathrm{m}$ \\
\hline Differenzdrehzahl & $n_{01 ; f}$ & $=30$ \\
\hline Festst of fdurchsatz & $m_{1}$ & $=300$ \\
\hline Rest feuchte & RF 1 & $=33.0$ \\
\hline ' & RF2 & $=39.2 \mathrm{Gem}-\mathrm{x}$ \\
\hline ' & AF3 & $=48.0 \mathrm{Gew}$ \\
\hline Nutzungsgrad & NG & $=73.4 x$ \\
\hline Fūllgrad & FG & $=1.6$ \\
\hline
\end{tabular}

Abb. 12: Rückstauprofil bei Herabsetzung der Rotordrehzahl

Den Nutzungsgrad kann man auch verbessern, indem man die Rotordrehzahl konstant hält, dafür aber die Differenzdrehzahl erhöht (Abb. 13). 
RÜCKSTAUPROF ILBERECHNUNG

(Der Start erfolgt am Austragsradius)

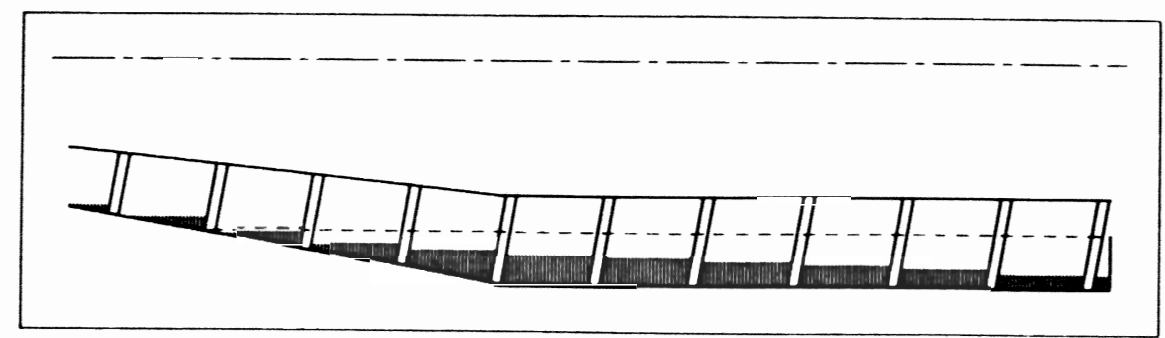

Maschinentyp: A

Zylinderdurchmesser $D_{2 y 1}=250 \mathrm{~mm}$

Austragsdurchmesser $D_{\text {wustr }}=165 \mathrm{~mm}$

Niveaudurchmesser $\quad D_{\mathrm{m} 2 \mathrm{v}}=190 \mathrm{~mm}$

Zylinderlánge $\quad L_{z y 1}=340 \mathrm{~mm}$

Konuswinkel $\quad B=10 \mathrm{Grad}$

Ganghöhe $G=55 \mathrm{~mm}$

Blattstărke $\quad D=6 \mathrm{~mm}$

Niveauvolumen $\quad V_{\text {miv }}=7.8 \mathrm{dm}^{3}$

\begin{tabular}{|c|c|c|}
\hline Rotordrehzahl & $n_{H}$ & $=30001 / \mathrm{min}$ \\
\hline Differenzdrehzahl & $n_{\text {oiff }}$ & $=35 \mathrm{1} / \mathrm{min}$ \\
\hline Feststof fdurchsatz & $m_{2}$ & $=300 \mathrm{~kg} / \mathrm{h}$ \\
\hline Restfeuchte & $\mathrm{AF} 1$ & $=33.0 \mathrm{Gew}-\mathrm{x}$ \\
\hline ' & AF2 & $=39.2 \mathrm{Gew}-\mathrm{x}$ \\
\hline ' & RF3 & $=48.0$ Gew $-\mathrm{x}$ \\
\hline Nutzungsgrad & NG & $=53.3 x$ \\
\hline Fūllgrad & $F G$ & $=3.2$ \\
\hline
\end{tabular}

Abb. 13: Rückstauprofil bei Erhöhung der Differenzdrehzahl

Bei solchen Überlegungen mu $B$ man natürlich bedenken, daß dabei andere unerwünschte Effekte auftreten können, so z.B. Störung der Klärung durch Turbulenzen bei Erhöhung der Differenzdrehzahl oder erschwerte Sedimentation bzw. höhere Restfeuchten beim Herabsetzen der Rotordrehzahl.

Die oben erwähnten Beispiele sind einzelne Betriebszustände. In der Abb.14a ist die Abhängigkeit des Nutzungsgrades NG vom Feststoffdurchsatz $\dot{m}_{S}$ bei verschiedenen Schleuderziffern $\mathrm{C}$ wiedergegeben.

Damit kann leicht beurteilt werden, für welche Betriebskombinationen der Dekanter noch relativ störungsfrei funktionieren kann. In der Abb. $14 \mathrm{~b}$ bzw. 14c ist ähnlich wie bei $14 \mathrm{a}$ die Abhängigkeit des Nutzungsgrades von der Differenzdrehzahl $\mathrm{n}_{\text {Diff }}$ bzw. vom Niveaudurchmesser $\mathrm{D}_{\mathrm{Niv}}$ bei Variation des C-Wertes zu sehen. 


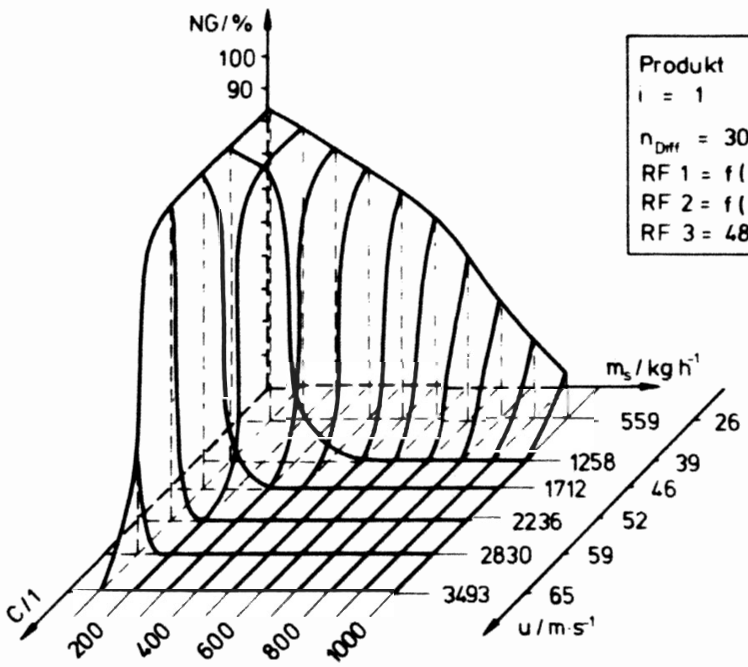

a)

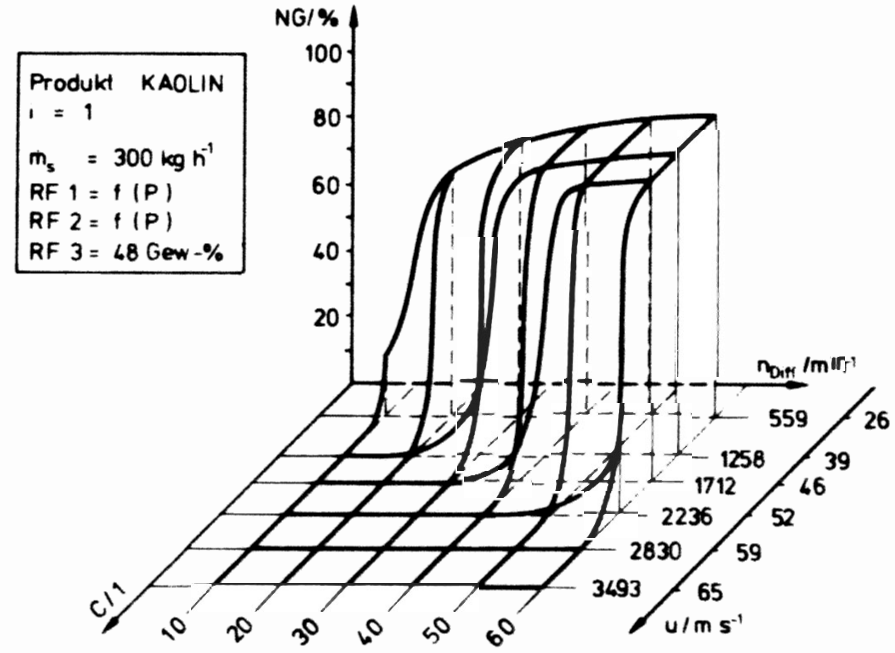

b)

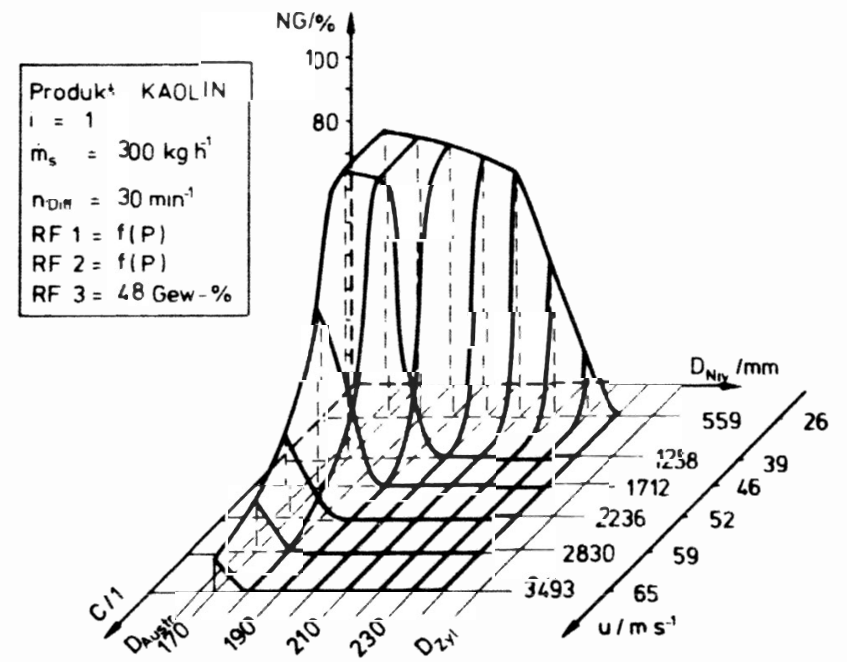

c)

Abb. 14: Betriebskennfelder zur Beurteilung der Funktionsfähigkeit des Dekanters 
4. Überlegungen zum "Scale-up"

Die Übertragung von Ergebnissen aus Pilotversuchen auf Großmaschinen (scale-up) ist, wie aus der Praxis bekannt, mit großen Unsicherheiten verbunden. So kann es z.B. vorkommen, daß die Großmaschine überhaupt keinen Feststoff austrägt, obwohl die Kleinmaschine relativ störungsfrei funktioniert hat. Dieser aus der Praxis schon bekannte Sachverhalt läßt sich auch theoretisch bestätigen und wird an einem Beispiel näher erläutert:

Nimmt man das Ergebnis aus der Abb. 12 als Beispiel für die Kleinmaschine, so sieht man, daß der Nutzungsgrad 73,4\% beträgt, d.h. der Dekanter würde bezüglich Feststoffaustrag und Klärung relativ gut funktionieren. Dies sieht man auch an dem Füllgrad, der nur 1,6 beträgt, d.h. das Sediment verweilt um den Faktor 1,6 länger in der Zentrifuge, als wenn diese rückstaufrei funktionieren würde. Beim "scaleup" werden die einzelnen Daten folgendermaßen verändert:

Modellausführung (M)

$\mathrm{C}_{\mathrm{M}}$

$\mathrm{n}_{\text {Diff, } M}$

$\dot{\mathrm{m}}_{\mathrm{Zulauf}, \mathrm{M}}$

$\mathrm{G}_{\mathrm{M}}$

$\mathrm{b}_{\mathrm{M}}$

${ }^{B} M$

$\mathrm{D}_{\mathrm{Zy}} \mathrm{l}, \mathrm{M}$

D Austrag, $M$

$\mathrm{D}_{\mathrm{NiV}, \mathrm{M}}$

Großausführung (G)

$=$

$=$

$=$

$=$

$=$

$=$

$=$

$=$

$=$
$\mathrm{C}_{\mathrm{G}}$

$\mathrm{n}_{\text {Diff, }}$

$\dot{\mathrm{m}}_{\mathrm{Zulauf}, \mathrm{M}} \cdot \mathrm{i}^{3}$

$G_{M} \cdot i$

$b_{M} \cdot i$

${ }^{B} \mathrm{G}$

$\mathrm{D}_{\mathrm{Zy}}, \mathrm{M} \cdot \mathrm{i}$

$D_{\text {Austrag }, M} \cdot i$

$\mathrm{D}_{\mathrm{Niv}, \mathrm{M}} \cdot \mathrm{i}$

Vergrößert man nun die Pilotmaschine (Abb.12) auf das 4-fache $(i=4)$, so kann man sehen, daß der Nutzungsgrad der Großmaschine $\mathrm{O} \frac{8}{8}$ beträgt (Abb. 15). 
RÜCKSTAUPROF ILBERECHNUNG

(Der Start erfolgt am Austragsradius)

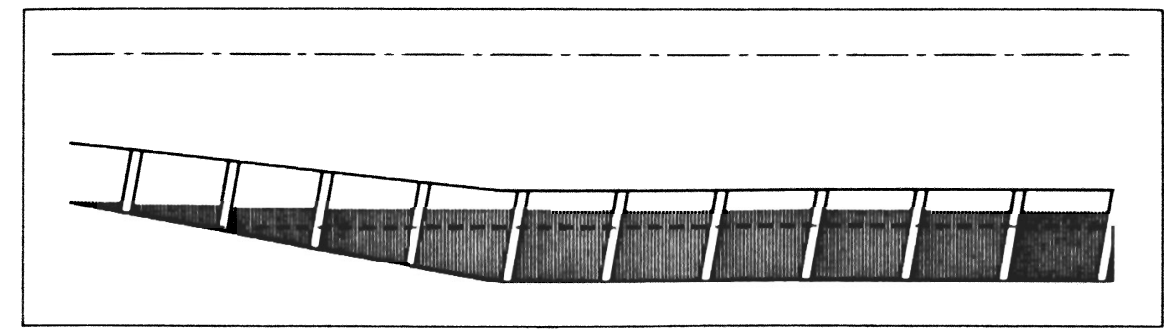

Maschinentyp : A $(4 x)$

Zylinderdurchmesser $D_{2 y 1}=1000 \mathrm{~mm}$ Austragsdurchmesser $D_{\text {Nustr }}=660 \mathrm{~mm}$ Niveaudurchmesser $\quad D_{\mathrm{Niv}}=760 \mathrm{~mm}$ Zylinder lănge $\quad L_{z y 1}=1360 \mathrm{~mm}$ Konuswinkel $\quad B=10 \mathrm{Grad}$ Ganghōhe $\quad G=220 \mathrm{~mm}$ Blattstärke $\quad D=24 \mathrm{~mm}$ Niveauvolumen $\quad V_{\text {miv }}=498.5 \mathrm{dm}^{3}$
Produkt : KAOLIN

\begin{tabular}{|c|c|c|}
\hline tordrehzahl & $n_{H}$ & $=13751 / n$ \\
\hline Differenzorehzanl & $n_{01 \% f}$ & 30 \\
\hline estst of fdurchsatz & $m_{s}$ & $=19200$ \\
\hline stfeuchte & $\mathrm{RF}_{1}$ & $=33.0$ \\
\hline ' & AF2 & $=39.2$ \\
\hline ' & RF 3 & $=48.0$ \\
\hline tzungsg & NG & $=0.0$ \\
\hline grad & $F G$ & 7 \\
\hline
\end{tabular}

Abb. 15: Rückstauprofil beim "scale-up"

In der $\mathrm{Abb}$. 15 erkennt man außerdem, daß die Rückstauhöhe am Zylinderende höher als das Flüssigkeitsniveau ist. Dies würde bedeuten, daß der Feststoff mit dem Zentrat abgezogen wird, bevor das sediment den Austragsradius erreicht hat, d.h. der Dekanter kann den Feststoff nicht austragen; die Klärung bricht völlig zusammen, die Suspension fließt ungetrennt in den Zentratablauf $a b$. Abb. 16 zeigt, daß der Einsatz eines Großdekanters praktisch nicht sinnvoll wäre, wenn beim "scale-up" die Schleuderziffer $C$ konstant gehalten wird. Die Großmaschine würde nur noch in einem sehr engen Bereich rückstaufrei funktionieren. 


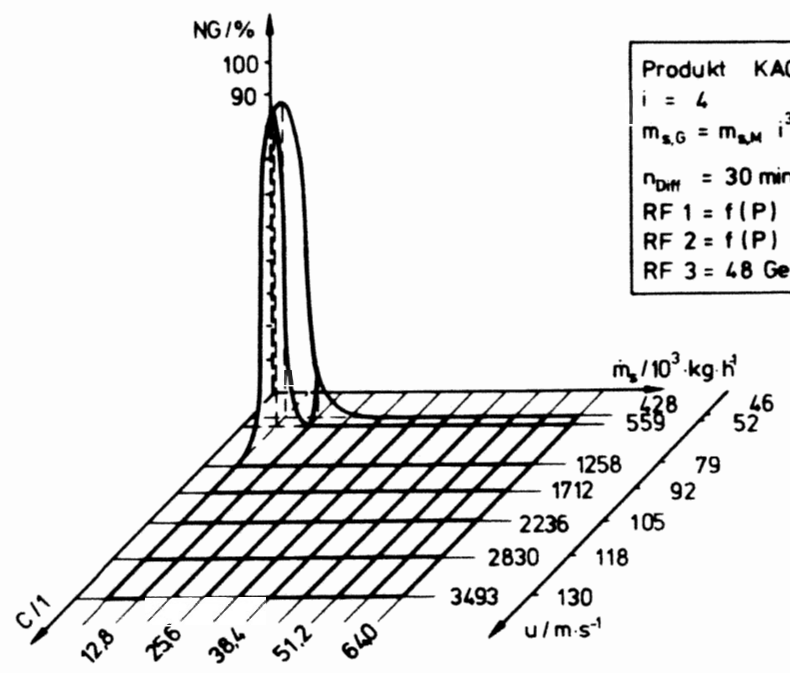

a)

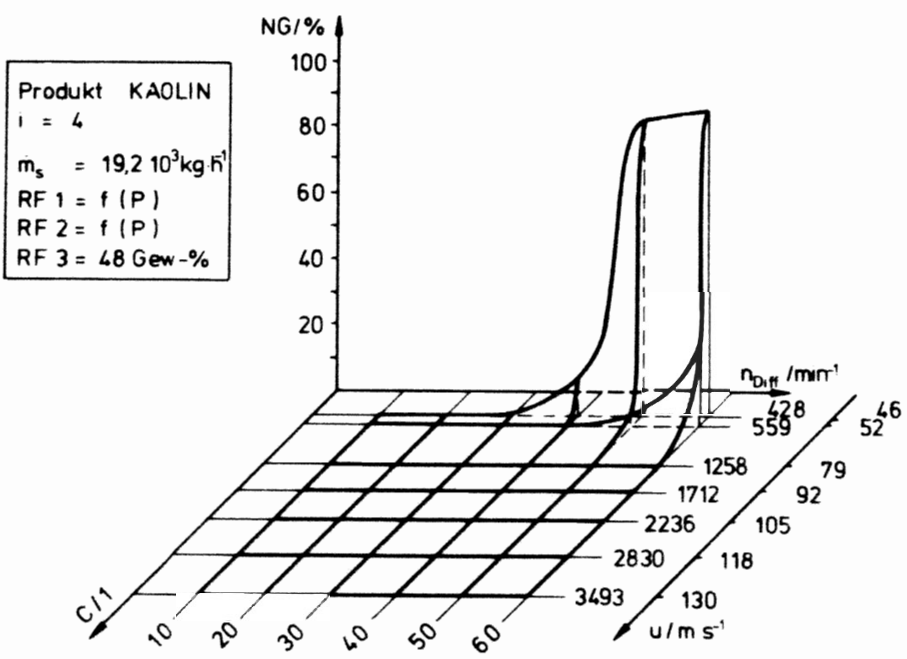

b)

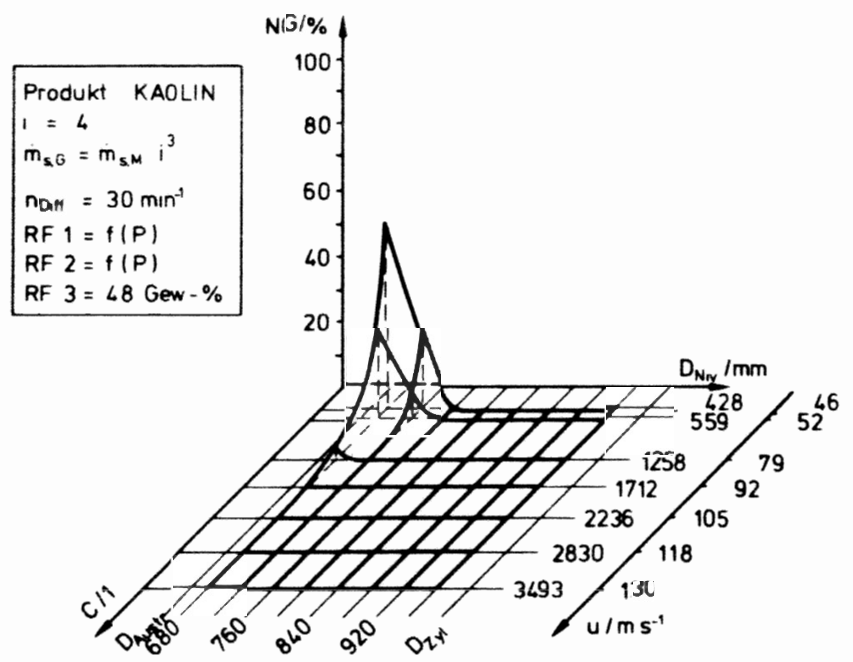

c)

Abb. 16: Betriebskennfelder beim "scale-up" $\left(\dot{\mathrm{m}}_{\mathrm{S}, \mathrm{G}}=\dot{\mathrm{m}}_{\mathrm{S}, \mathrm{M}} \cdot \dot{i}^{3}\right)$ 
Vergleicht man $\mathrm{Abb} .16$ mit $\mathrm{Abb} .14$, so erkennt man, daß die Großmaschine bezüglich des Rückstaus sich ähnlich verhalten würde, wenn beim "scale-up" nicht die Schleuderziffer C sondern die Umfangsgeschwindigkeit u der Trommel konstant gehalten würde. Dies kann auch durch Ähnlichkeitsbetrachtung aus Gl. (30) hergeleitet werden. Für die Schleuderziffer $C$ gilt demnach: $C_{G}=C_{M} / i$.

Wenn man nun den Feststoffdurchsatz $\dot{\mathrm{m}}_{\mathrm{s}}$ nicht nach dem Schleppkraftansatz $|3| \mathrm{mit}^{3}$ vergrößert, sondern nach Stokes mit $i^{2}$, so wird deutlich, daß bei konstanter Umfangsgeschwindigkeit die Großmaschine sogar günstiger arbeiten würde als die Pilotmaschine (Abb. 17).

Wie schon erwähnt, wurden die hier aufgeführten Beispiele unter der Annahme einer festen Grundschicht gerechnet. Für den Fall, daß das sediment neben der spiraligen Rückströmung auch durch den Spalt zwischen Schneckenblatt und Konuswand zurückfließen kann, wird das Rückstauprofil noch ungünstiger. Hinzu kommt noch, daß es bei gleichzeitigem Rückfließen des Schlamms durch den spalt und in spiraliger Richtung - je nach Oberflächenrauhigkeit - auch das Gleiten der Paste an den Metalloberflächen berücksichtigt werden muB.

Bei der Herleitung dieses Rechenmodells bzw. bei der Berechnung der Rückstauprofile wurden gewisse Annahmen getroffen, wodurch mit entsprechender Vorsicht auch quantitative Aussagen gemacht werden können. Mit dem Rechenmodell ist man jetzt viel besser in der Lage, gute Relativaussagen zu treffen, vor allem dann, wenn man das Versuchsergebnis einer Pilotmaschine auf eine Großmaschine übertragen möchte. Kritisch zu sehen ist der große experimentelle Aufwand zur Bestimmung der rheologischen Daten $\left(\tau_{0}, k, n, \tau_{1}, c, m\right)$, die fortlaufend von der Restfeuchte des Sediments abhängen. Da die Änderung der Schlammrestfeuchte während der Schneckenförderung nicht bekannt ist, wurde sie in erster Näherung als linear angenommen. Man könnte jedoch davon ausgehen, 


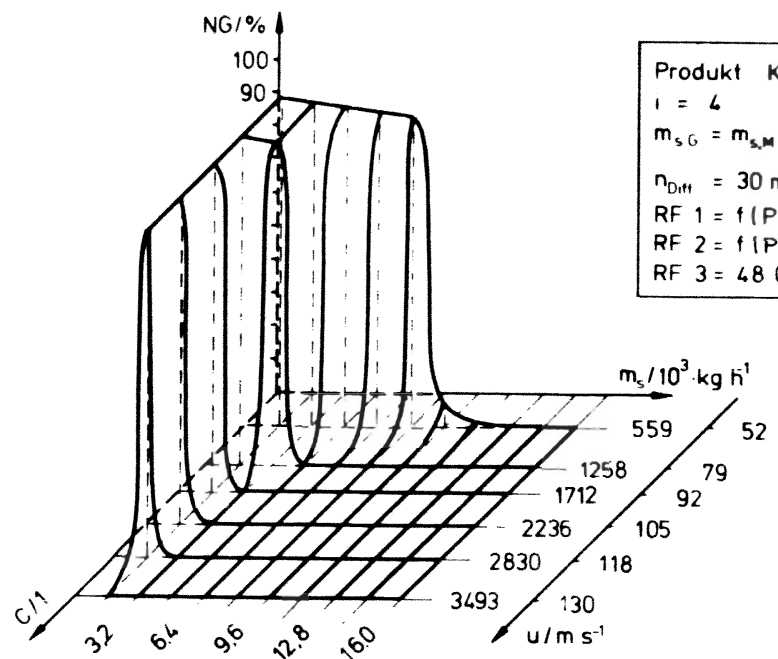

a)

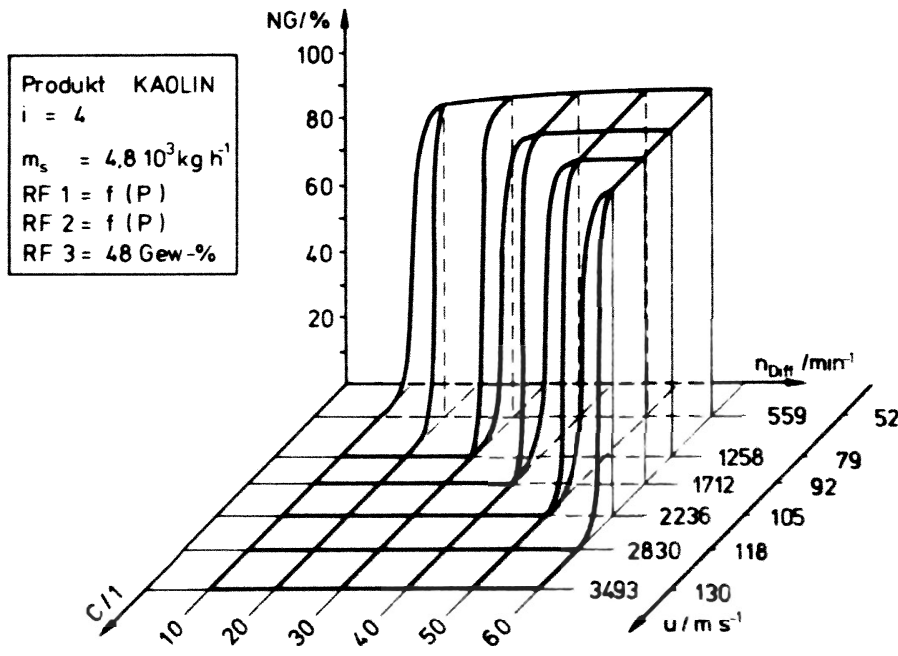

b)

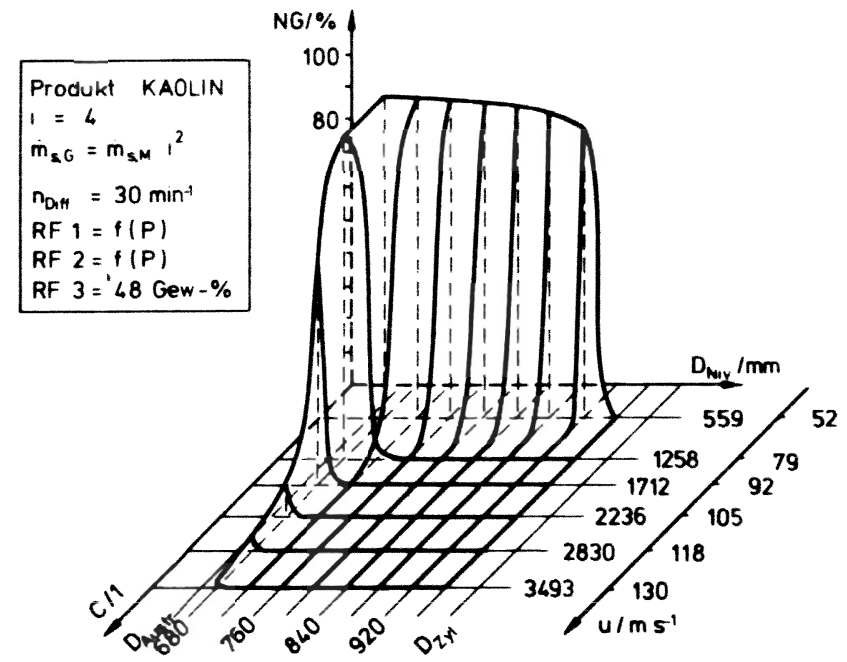

C)

Abb. 17: Betriebskennfelder beim "Scale-up" $\left(\dot{\mathrm{m}}_{\mathrm{S}, \mathrm{G}}=\dot{\mathrm{m}}_{\mathrm{S}, \mathrm{M}} \cdot \dot{i}^{2}\right)$ 
daß sich die Restfeuchte im konischen Bereich des Dekanters durch die Abnahme der Zentrifugalbeschleunigung nicht oder kaum mehr ändert, so daß die Werte für RF1 und RF2 für die Rechnung praktisch konstant gehalten werden können.

Das hier beschriebene Rechenmodell wurde auch anhand experimenteller Daten aus einem industriellen Einsatzfall überprüft. Bei diesem Einsatzfall wurden zur Behandlung von Stärkesuspension zwei Dekanter unterschiedlicher Geometrie eingesetzt. Dabei hat man festgestellt, daß die größere Maschine den Feststoff überhaupt nicht austrug. Nach Ermittlung der rheologischen Daten des Produkts konnte dieser experimentelle Befund auch rechnerisch nachgewiesen werden.

\section{Formelzeichen}

$\begin{array}{ll}\text { b } & \text { Blattstärke } \\ \text { C } & \text { C-Wert, Schleuderziffer } \\ C^{\prime} & \text { Gleitkonstante } \\ \text { FG } & \text { Fülgrad } \\ \text { g } & \text { Beschleunigung } \\ \text { G } & \text { Ganghöhe } \\ \text { h } & \text { Kuchenhöhe, Stauhöhe } \\ \text { i } & \text { Vergrößerungsfaktor, scale-up-Faktor } \\ \text { K } & \text { Fließkonstante } \\ \text { m } & \text { Gleitindex } \\ \text { m } & \text { Feststoffmassenstrom } \\ n & \text { Fließindex } \\ n_{D i f f} & \text { Differenzdrehzahl } \\ n_{H} & \text { Rotordrehzahl } \\ \text { NG } & \text { Nutzungsgrad } \\ \text { p } & \text { Druck } \\ \text { P } & \text { Pressung } \\ \text { R } & \text { Radius } \\ \text { RF } & \text { Restfeuchte } \\ \text { u } & \text { Umfangsgeschwindigkeit } \\ v_{g} & \text { Gleitgeschwindigkeit } \\ \end{array}$




$\begin{array}{ll}\dot{\mathrm{V}} & \text { Volumenstrom } \\ \mathrm{x}_{50} & \text { mittlerer Partikeldurchmesser } \\ \alpha & \text { Schneckensteigungswinkel } \\ B & \text { Konuswinkel } \\ \dot{\gamma} & \text { Schergradient } \\ \delta & \text { Verschneidungswinkel } \\ \rho & \text { Dichte } \\ \bar{\rho} & \text { mittlere Sedimentsdichte } \\ \rho_{1} & \text { Flüssigkeitsdichte } \\ \tau & \text { Schubspannung } \\ \tau_{0} & \text { Fließgrenze, Anfangsschubspannung } \\ \tau_{1} & \text { Gleitgrenze } \\ \tau_{W} & \text { Wandschubspannung }\end{array}$

\section{Schrifttum}

|1| A. Karolis, W. Stahl: Proc. Symp. Solids/Liquids Separation Practice and the Influence of new Techniques, Univ. of Leeds, England, 2.-5. April 1984

|2| E. Windhab, W. Gleißle: Advances in Rheology, Vol.2: Fluids Proc. IX. Int. Congress on Rheology, Mexico, 1984 , p. 557-564

|3| W. Stahl, T. Langeloh: Ger. Chem. Eng. 7 (1984) $72-84$ 


\section{Anhang}

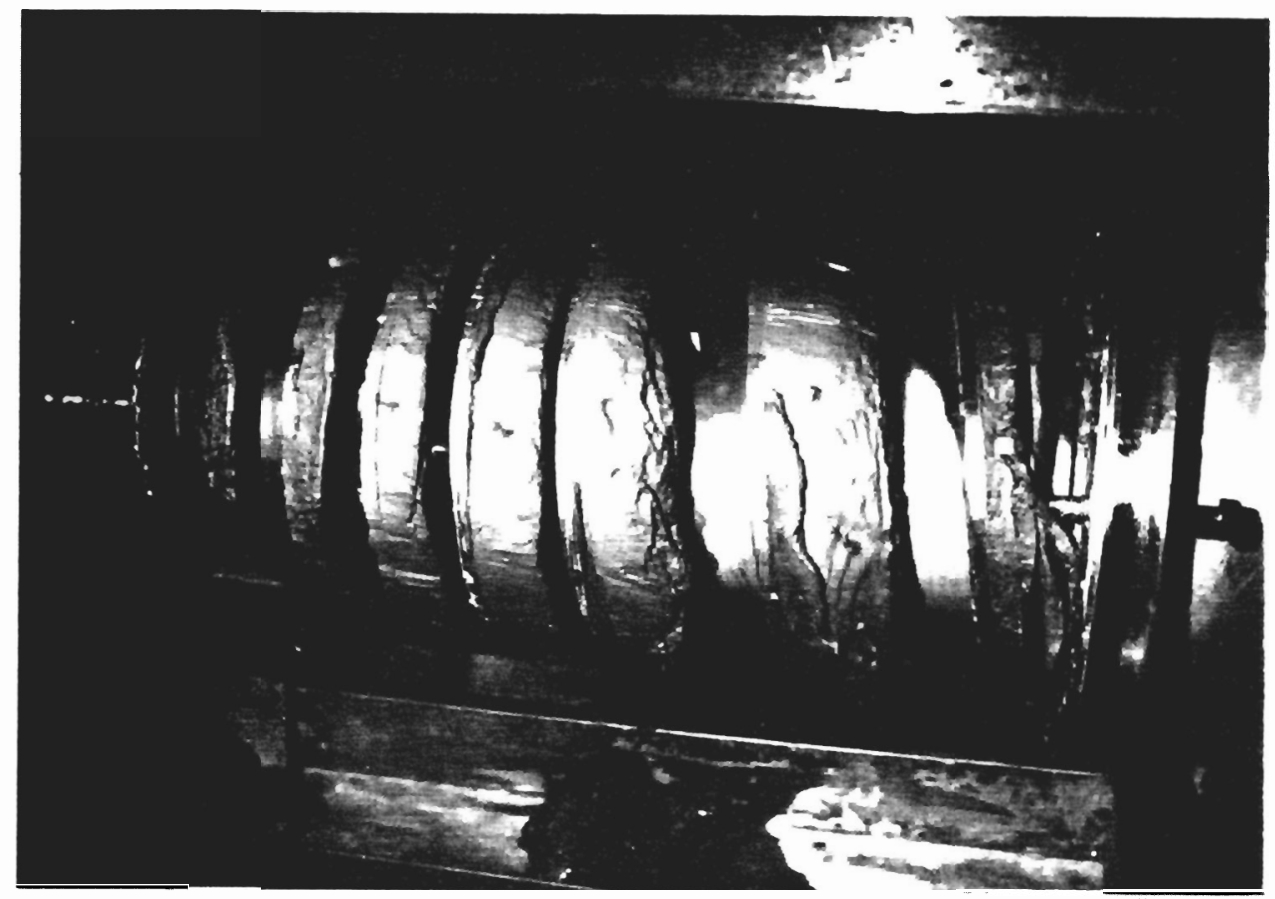

Abb. 3: Schlammrückstau in einem ausgebauten Dekanter (Durch das Herausziehen der Schnecke streift sich der deponierte Feststoff ebenso wie die Grundschicht auf der Rückseite der Schneckenblätter ab. Die Formation des Kuchens entspricht deshalb nicht dem Zustand im Betrieb.) 